\title{
Stochasticity and controllability of nutrient sources in foraging: host-feeding and egg resorption in parasitoids
}

\author{
ROMAIN RICHARD ${ }^{1}$ AND JÉRÔME CASAS ${ }^{2}$ \\ Université de Tours, Institut de Recherches sur la Biologie de l'Insecte, IRBI UMR CNRS 6035, Av. Monge, 37200 Tours, France
}

\begin{abstract}
The trade-off between current and future reproduction has led many organisms experiencing stochastic reproductive opportunities to be flexible in their resource acquisition and allocation rules. Many parasitoid wasps display flexibility in choosing to host-feed or oviposit on a host and possess an ovarian system enabling nutrient reallocation through egg resorption.

The aim of this work is to assess the complementary adaptive values of host-feeding and egg resorption as functions of host density in a synovigenic (maturing eggs throughout its adult life) parasitoid, Eupelmus vuilleti (Hymenoptera: Eupelmidae), for which there is a uniquely large base of relevant knowledge. We developed a series of models of increasing complexity, starting from a simple analytical model without egg resorption and moving on to data-rich stochastic dynamic programming models (SDP), without and with resorption.

The analytical model enabled the characterization of two, long- and short-term, foraging strategies which determine host usage. Oviposition is favored at low host densities (leading to the short-term strategy), while host-feeding is favored at high host densities (leading to the long-term strategy). The change of strategy occurs abruptly at intermediate host densities. The SPD models not only confirmed these predictions, but also identified smaller regions of decisions driven by day/night cycles and approaching death and predicted major shifts in daily activity patterns according to the chosen strategy. The fitness gain due to resorption is highest at intermediate host densities, where females adopt the riskier but more profitable long-term strategy. Such a result contrasts with the generally held view, which assumes highest gains at the lowest host densities. A counterintuitive result is the higher prevalence of host-feeding associated with the ability to resorb eggs.

Considering egg resorption as a last-resort strategy is underestimating its adaptive value, which is best understood with reference to other sources of nutrients. Its deterministic and controllable nature acts as insurance to forage and oviposit at low host densities, despite irregular food availability and potential death through starvation. Thus timing, not so much overall energy gain, matters in egg resorption. The approach can be extended to other situations, and we highlight an unexpected analogy of our results with the hoarding behavior of vertebrates.

Key words: capital and income resources; energy management; hoarding; host handling; life-history strategies; mixed diet; oosorption; optimal foraging; resource allocation; resource acquisition; stochastic dynamic programming; synovigeny.
\end{abstract}

\section{INTRODUCTION}

The existence of a trade-off between immediate and future reproduction is one of the key elements in the theory of life-history traits (Williams 1966, Roff 1992, Stearns 1992, Roff and Fairbairn 2008). As organisms have a limited amount of resources to allocate among functions, investment in immediate reproduction is made at the expense of other functions, including survival and future reproduction. Life histories emerge from the way individuals manage their resource

\footnotetext{
Manuscript received 21 August 2008; revised 5 January 2009; accepted 8 January 2009. Corresponding Editor: S. J. Simpson.

${ }^{1}$ Present address: Ecology Division, Department of Biological Sciences, University of Calgary, Calgary, Alberta, Canada T2N $1 \mathrm{~N} 4$.

${ }^{2}$ Corresponding author.

E-mail: jerome.casas@univ-tours.fr
}

allocation toward these different functions. Some allocation strategies make a greater contribution to reproductive success than others, and natural selection should favor the strategies most likely to increase the reproductive success (Houston and McNamara 1999). The trade-off between immediate and future reproduction plays a particularly important role in decision making in organisms, such as many parasitoids, for which the food source is also the site of reproduction. Host-feeding favors future reproduction due to the acquisition of nutrients. Depending on the species concerned, this benefit may result in the production of more eggs, greater longevity, or both (Jervis and Kidd 1986, Collier 1995a, Heimpel and Collier 1996, Heimpel et al. 1997, Giron et al. 2004). Conversely, oviposition results in an immediate gain in fitness. Nutrition and reproduction are often incompatible in these circum- 
stances, as host-feeding may kill the host or reduce its quality such that it cannot be used for oviposition (Jervis and Kidd 1986). We do not deal here with concurrent host-feeding and oviposition on the same host, a phenomenon which occurs for some species (Rivero and West 2005). Hymenopteran parasitoids have thus become the model of choice for studies of behavior relating to this trade-off (Price 1973, Charnov and Stephens 1988, Godfray 1994, Rivero and Casas 1999a, Jervis et al. 2008). Many parasitoids have food sources away from the host as well (e.g., nectar, pollen, and the like), but we will not deal any further with these cases.

The costs and benefits associated with host-feeding and oviposition vary with the physiological state of the individual (e.g., energy reserves, egg load, age) and the characteristics of the environment in which that individual finds itself (e.g., host availability and quality, extrinsic mortality). When females encounter a host, they adjust their behavioral response as a function of these variables to maximize their reproductive success. Egg load has been identified, both theoretically and empirically, as one of the essential factors determining whether a female decides to feed on a host (Mangel 1989, Minkenberg et al. 1992, Chan and Godfray 1993, Collier 1995b, Heimpel and Rosenheim 1995, Heimpel and Collier 1996, Heimpel et al. 1998). Host-feeding is generally favored by a decreasing egg load, as it enables females to reduce the risk of egg limitation in the short term (as the eggs are not used for oviposition) and in the longer term, as it may also make it possible to increase egg production in the more distant future (Heimpel et al. 1996, Rivero and Casas 1999b). Models have also predicted that host-feeding would be favored by a decrease in energy reserves (Chan 1991, Houston et al. 1992, Chan and Godfray 1993, Collier 1995b, Heimpel and Collier 1996, Heimpel et al. 1998, Burger et al. 2004). Host-feeding may thus allow the female to reduce the risks of egg and/or time limitation, depending on the final destination of the nutrients. In the ideal situation, a female laying her last egg as she dies achieves the perfect balance of investment in eggs vs. longevity. However, encounters with hosts are stochastic, to such an extent that this ideal is highly unlikely ever to be realized (Rosenheim 1999a, Ellers et al. 2000, van Baalen 2000, Jervis et al. 2001). The aim of simultaneously limiting the impact of time and egg limitations on reproductive success in a stochastic environment necessitates a high level of flexibility in resource acquisition and allocation.

The balance between costs in eggs and costs in time is more readily reached if the organism can make decisions about the allocation of at least some of the nutrients acquired. The optimization of resource allocation as a function of the conditions experienced requires the use of a set of indicators based on environmental and physiological signals (reviewed by Papaj 2000). The dynamic nature of energy allocation and egg production allows the rapid adjustment of resource allocation to immediate and future reproduction according to the conditions encountered (Rivero and Casas 1999a, Papaj 2000, Casas et al. 2009). Indeed, insect ovaries have adapted to adjust egg production rapidly: ovarian dynamics are regulated by a process of egg maturation known as oogenesis and, in many species, by a process of egg destruction known as resorption (Flanders 1942, Bell and Bohm 1975, Eckelbarger 1994, Wheeler 1996, Papaj 2000). Resorption has a particular effect on energy and ovarian dynamics, allowing insects to reallocate nutrients initially invested in reproduction to other functions, through egg destruction (Bell and Bohm 1975). Resorption occurs in many insect taxa (reviewed by Bell and Bohm 1975). It is currently seen as a "lastresort" strategy in parasitoids, due to the major costs it entails (Jervis et al. 2001, Bernstein and Jervis 2007). Nonetheless, the real consequences of this phenomenon for the reproductive success and life history of individuals remain largely hypothetical.

In hymenopteran parasitoids, resorbed eggs are the terminal, mature ones (Flanders 1942, Jervis and Kidd 1986). This process entails large energy costs, associated with the initial construction of a mature egg and its subsequent destruction. Furthermore, as the destroyed egg can no longer be laid, the female is also at risk of oosorption-mediated egg limitation, in which the number of eggs available for oviposition is reduced by resorption (Rosenheim et al. 2000). These costs have been well characterized, but not quantified. The associated benefits, by contrast, are even harder to identify (Asplen 2007). Resorption occurs mostly in individuals subjected to periods of nutritive stress, to the extent that it is normally seen as a source of additional energy allowing the animal to survive (Grosch 1950, King and Hopkins 1963, Bell and Bohm 1975). However, Casas et al. (2005) have called into question the importance of the quantitative contribution of resorption to survival, as an egg contains enough energy to cover $<10 \%$ of daily metabolic requirements of the parasitoid. Nonetheless, Rosenheim et al. (2000) have pointed out that prolonging life, even by only a few hours, may result in a major gain of fitness if it allows female to survive for long enough to experience periods of high host availability.

Resorption also occurs in other situations (RiveroLynch and Godfray 1997, Gauthier and Monge 1999, Asplen 2007). This process may be triggered by an agedependent destruction mechanism, to replace the oldest and potentially least viable eggs by newly matured eggs (Rivero-Lynch and Godfray 1997). Alternatively, resorption may maintain a continuous egg production process in the absence of hosts (as oviposition would normally create the space for additional eggs and this process does not occur in the absence of hosts). The nutrient recycling accompanying resorption is an economic way to achieve this objective (Flanders 1942, King and Richards 1968, Jervis and Kidd 1986). This hypothesis does not necessarily exclude the possibility of a role in survival, and resorption may be seen as a 
mechanism for adjusting egg production to the conditions encountered by the individual, making use of the nutrients invested in the oocytes (King and Richards 1968, Bell and Bohm 1975, Papaj 2000). We have included resorption in a model based principally on energy considerations. This precludes testing of the hypothesis of an age-dependent degeneration mechanism, which would require physiological studies. It does make it possible, however, to estimate in which way ovaries respond to variations in foraging success and oviposition activities when bidirectional energy allocation is enabled.

Resorption and host-feeding present strong similarities, in that both favor future reproduction and both supply nutrients for survival and/or egg production. Both are detrimental to immediate reproduction, except in the limiting cases where a female is eggless or the host unsuitable for oviposition, as a potential host is sacrificed during host-feeding and an egg that could have been laid is destroyed during resorption. These costs raise questions about the conditions under which it is advantageous to sacrifice a host or an egg for the good of future reproduction. In other words, at what point is the gain associated with host-feeding or resorption greater than the cost associated with the missed opportunity to lay an egg on an existing (in the case of host-feeding) or potential (in the case of resorption) host? As for the classic trade-off between host-feeding and oviposition on hosts, there is a clear trade-off in the use of mature eggs: should the egg be retained for subsequent laying or should it be resorbed? The simultaneous presence of several eggs in the ovaries makes it difficult to quantify the costs and benefits of the various components of this trade-off. Furthermore, the resorption of an egg is not necessarily equal to the loss of an oviposition. The relationship between resorption and host-feeding is clearly complex, even if both types of behavior address the same fundamental problem. The similarities and differences between these types of behavior raise questions about how the insect decides what to do and when. The aim of this study was to evaluate the adaptive value of host-feeding and resorption and the links between these two processes, through two quantitative approaches - one analytical and the other computational. Thus, the focus of our work is on both the adaptive value of resorption and the complementary roles of hostfeeding and resorption on foraging behavior. As this covers already a large territory of the unknown, we simplified our task in assuming constant host quality, nonconcurrent host use for reproduction and nutrition, no access to other sources of nutrient, host acceptance upon encounter, and time-invariant probability of host encounter.

We used Eupelmus vuilleti (Crawford) (Hymenoptera: Eupelmidae) as our model system (see Plate 1). This parasitoid is a synovigenic (maturing eggs throughout its adult life) host-feeder. It will feed on a sugar solution if offered, but otherwise lives in granaries where carbohy- drate substrates are not present. A large amount of data is available concerning its energy dynamics, allowing a fine and highly realistic model to be developed (Giron et al. 2002, Giron and Casas 2003a, b, 2004, Casas et al. 2005). An analysis of the underlying biological phenomena likely to condition decisions concerning hostfeeding, the better understood of the two processes considered, was carried out with an analytical model. We were then in a position to accurately interpret the results of a highly realistic, but much more complex model of the optimization of dynamic programming, with and without resorption. Based on the conclusions drawn about host-feeding and analogies with resorption, we were able to identify the situation favoring resorption. Finally, we placed these two processes for the acquisition and allocation of resources into a broader context of life history traits and behavioral ecology, accounting for differences and similarities between these strategies and other foraging strategies in other organisms.

\section{Models}

We first describe the construction of models, starting from a simple analytical model of host-feeding and working toward the stochastic dynamic programming (SDP) model. We then describe the estimation of parameter values. As stated in the Introduction, both the analytical models and the two variants of the SDP model assume constant host quality, nonconcurrent host use for reproduction and nutrition, no access to other sources of nutrient, host acceptance upon encounter, and time-invariant probability of host encounter. A description of state variables and parameters used in the models is given in Table 1.

\section{Analytical model of host-feeding}

We identified the conditions under which host-feeding is beneficial by constructing an analytical model based on simple hypotheses. Let us first consider a situation in which the female parasitoid has only one opportunity to feed. When this parasitoid encounters its first host, it can choose to feed or to lay an egg. The use of the host for feeding constitutes a lost opportunity for oviposition. Host-feeding can thus only improve fitness if this loss of an opportunity for oviposition is compensated for by an additional oviposition made possible by the prolongation of life achieved by host-feeding. In order to kept the assumptions as few and simple as possible, this model does not keep track of the egg load, and hence nor of the possibility of egg limitation. So, host-feeding cannot directly increase fecundity. However, an increase in fecundity is indirectly reached, concomitantly to the prolongation of life. Furthermore, hosts are encountered at a fixed rate, thus excluding any source of stochasticity in host encounter. Cycling between day and night is not taken into account. Finally, a female parasitoid feeds only if the total number of hosts encountered in her life, accounting for the prolongation of life achieved by an host-feeding, minus one (oviposition loss due to host- 
TABLE 1. Definitions of all variables and parameters used in the text for the analytical model and the dynamic programming models. Data on the biology of the hymenopteran parasitoid Eupelmus vuilleti are also included.

\begin{tabular}{|c|c|c|c|}
\hline Parameter & Description & Value & References \\
\hline$H_{\mathrm{a}}$ & probability of host encounter in one time step & varied & \\
\hline$D y$ & $\begin{array}{l}\text { number of time steps a female can survive with } \\
\text { one egg equivalent }\end{array}$ & 8.77 & inferred \\
\hline$\lambda$ & probability of host encounter in one time step & varied & \\
\hline$\mu$ & probability of extrinsic mortality in one time step & 0.0003 & inferred \\
\hline$\Delta t$ & length of the time step in SDP (minutes) & 10 & \\
\hline$t_{\mathrm{a}}$ & number of time steps during the active period & 36 & \\
\hline$t_{\mathrm{n}}$ & number of time steps during the inactive period & 108 & \\
\hline$X_{\max }$ & maximum egg load & 12 & $\begin{array}{l}\text { R. Richard (personal } \\
\text { observation) }\end{array}$ \\
\hline$Y_{\max }$ & maximum energy reserves (egg equivalents) & 120 & Casas et al. (2005) \\
\hline$Y_{\mathrm{m}}$ & $\begin{array}{l}\text { energy used for maintenance in one time step } \\
\text { (egg equivalents) }\end{array}$ & 0.114 & inferred \\
\hline$Y_{\mathrm{h}}$ & gained energy from host-feeding (egg equivalents) & 5.22 & Casas et al. (2005) \\
\hline$\alpha$ & number of eggs matured in one time step & 0.02 & inferred \\
\hline$D$ & maximum life span (days) & 26 & Casas et al. (2005) \\
\hline$R_{\max }$ & maximum number of resorbing eggs & 12 & assumption \\
\hline$\delta$ & proportion of each egg resorbed in one time step & 0.01 & assumption \\
\hline$\gamma$ & conversion efficiency of resorption and host-feeding & 0.7 & McDonald et al. (2002) \\
\hline \multicolumn{4}{|c|}{ Variables for SDP model } \\
\hline$t$ & time step number during active period & $1, \ldots t_{\mathrm{a}}$ & \\
\hline$d$ & day number of life & $1, \ldots D$ & \\
\hline$y(t, d)$ & energy reserves (egg equivalents) & $\left(0, Y_{\max }\right)$ & \\
\hline$x(t, d)$ & number of mature eggs (egg load) & $\left(0, X_{\max }\right)$ & \\
\hline$r(t, d)$ & number of resorbing eggs & $\left(0, R_{\max }\right)$ & \\
\hline$i$ & host-handling decision & 1,2 & \\
\hline$k$ & number of eggs put into resorption & $0, \ldots R_{\max }$ & \\
\hline \multicolumn{4}{|c|}{ Biology of Eupelmus vuilleti } \\
\hline \multirow[t]{5}{*}{$n_{\mathrm{h}}$} & mean life span (days) & $14.25 \pm 4.18$ & Casas et al. (2005) \\
\hline & lifetime mean number of ovipositions & $39 \pm 17.6$ & Casas et al. (2005) \\
\hline & lifetime mean number of host-feeding events & $21.7 \pm 10.86$ & Casas et al. (2005) \\
\hline & mean number of ovarioles & 6 & $\begin{array}{l}\text { R. Richard ( personal } \\
\text { observation) }\end{array}$ \\
\hline & mean length of time to host-feed (minutes) & $17.30 \pm 0.84$ & Giron et al. (2004) \\
\hline
\end{tabular}

Notes: The term "inferred" means that these values are obtained from experimental data, after some calculations. "Assumption" refers to the case where values were estimated using plausible values from the literature of other species (see Model: Estimation of parameters for more explanation). Blank cells indicate that the values were set by the authors. SDP refers to stochastic dynamic programming models.

feeding, left-hand side of Eq. 1), is greater than the total number of hosts she would have encountered in her life otherwise (right-hand size of Eq. 1). The gains from oviposition and host-feeding are therefore equivalent when

$$
\begin{gathered}
\left(Y+Y_{\mathrm{h}}\right)(D y)(1-\mu)^{\left(Y+Y_{\mathrm{h}}\right) D y}\left(H_{\mathrm{a}}\right)-1 \\
=Y(D y)(1-\mu)^{Y D y}\left(H_{\mathrm{a}}\right)
\end{gathered}
$$

with $Y$ the initial level of energy reserves, $Y_{\mathrm{h}}$ the energy gained from an host-feeding, $D y$ the number of time steps that the female can live with one energy unit, $H_{\mathrm{a}}$ the probability of host encounter in one time step, and $\mu$ the probability of extrinsic mortality in one time step (Table 1).

For comparison with the SDP model, in which the probability of encountering a host in each time step is denoted $\lambda$, it should be pointed out that when

$$
\lambda=\frac{t_{\mathrm{a}}}{t_{\mathrm{a}}+t_{\mathrm{n}}} H_{\mathrm{a}}
$$

females from analytical and SDP models encounter the same mean number of hosts per day. The variable $t_{\mathrm{a}}$ represents the number of time steps during which the female is active and encounters hosts and $t_{\mathrm{n}}$ represents the number of time steps in the period of inactivity during which the female does not encounter hosts. We then also use the parameter $\lambda$ to express the probability of host encounter in the analytical model, using the equivalence just stated. This system provides the $(\lambda, Y)$ pair for which the two strategies are equivalent, dividing the space into two parts: one in which host-feeding is beneficial and the other in which it is disadvantageous and egg laying is preferable.

We then analyzed an additional compromise likely to condition host-feeding by counterbalancing the risk of death due to starvation by the risk of extrinsic mortality (due to predation, abiotic factors, and the like). There is an energy threshold at which the female has the same chances of dying from each of these two causes. This threshold is given by resolving the following equation:

$$
\left(1-H_{\mathrm{a}}\right)^{Y D y}=1-(1-\mu)^{Y D y} .
$$


The term at the left of the equation represents the probability of not encountering a host before the exhaustion of energy reserves, given that the female has $Y$ energy units available. The term at the right of the equation represents the probability of dying from another cause during the same period of time. The result of this equation divides the $(\lambda, Y)$ space into two, with the female more likely to die from starvation in one part and more likely to die from extrinsic causes in the other. By combining the results of the two equations of the analytical model, we obtain a space $(\lambda, Y)$ divided into four parts, in each of which different compromises are likely to operate (see Results).

\section{Dynamic programming models}

Here we aim to describe the construction of the dynamic programming model. We describe the principles underlying the construction of a model without resorption and then explain how resorption is added. The scheme of the model is shown in Fig. 1. Then, the fundamental equations of the model with resorption are given (Models: Dynamic programming models: Equations of the model). Once dynamic equations are solved backward, it is possible to implement Monte Carlo forward simulations, as described later on (Models: Dynamic programming models: Forward iterations).

Construction principles.-The model without resorption was constructed as follows:

1) The progression of the parasitoid over time is represented as following: each day $d$ is composed of a period of active searching for hosts and a period of inactivity. The active period is divided into a discrete sequence of intervals $t$, of length $\Delta t$ (10 minutes), during which the parasitoid searches for hosts. The active period counts $t_{\mathrm{a}}$ time steps, the inactive period $t_{\mathrm{n}}$ time steps. The maximum potential life span of the organism is $D$ days. The active period is analyzed for every time step $(t, d)(t=1-36 ; d=1-26)$ between emergence and maximum life span $(36,26)$. The whole night is discounted for in a single operation (sequential coupling, Clark and Mangel 2000) covering $t_{\mathrm{n}}$ time steps, starting from the last time step of the active period considered $(36, d)$, and ending the first time step of the active period of the following day $(1, d+1)$.

2) At each time step, the organism is described by two dynamic variables: $X(t, d)$, the mature egg load, and $Y(t, d)$, the energy reserves at the same time step. When energy levels fall to a certain threshold, $Y_{\text {crit }}$, the organism dies. The energy acquired by host-feeding beyond a certain threshold, $Y_{\max }$, and the number of eggs produced beyond $X_{\max }$ are considered lost.

3) Changes in these variables depend on the behavioral decisions taken and behaviorally independent physiological parameters: the metabolic energy costs, grouped together in the term $Y_{\mathrm{m}}$, and the number of eggs matured in one time step, $\alpha$, produced from energy reserves (Fig. 1). The parameters of the model associated

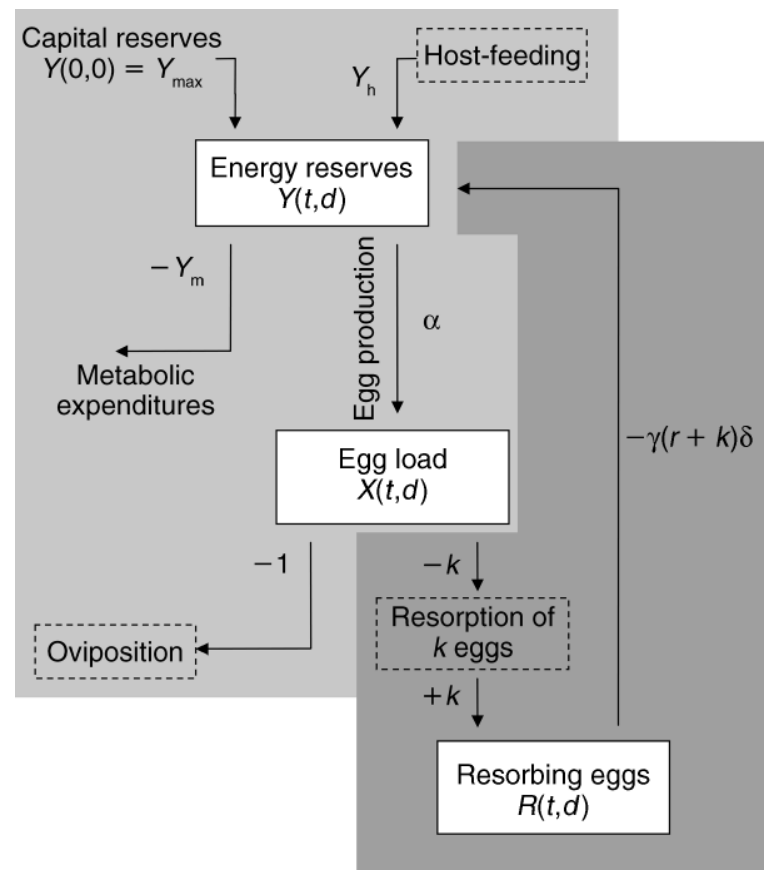

FIG. 1. Relationship between state variables of the stochastic dynamic program without (light gray area) and with egg resorption (light and dark gray areas) in a parasitoid. Variables are boxed with solid lines, and decisions are represented with dashed lines. See Table 1 for the definitions of variables.

with the environment explored by the females are stochastic: the probability of extrinsic mortality in one time step, $\mu$, and the probability of host encounter in one time step, $\lambda$.

4) When the female encounters a host, she must choose between $(i=1)$ host-feed or $(i=2)$ lay an egg. We then calculate the expected reproductive value for each decision. This value corresponds to the sum of the total number of descendants produced at the time step starting at $(t, d)$, assuming that the organism takes decision $i$, and the expected number of descendants during the rest of its life, assuming that the organism subsequently behaves in an optimal manner. The program did calculate the fitness associated with rejection. Rejection appears to occur only as a rare case, when reserves are full and females have no eggs to lay when encountering a host, what occurs mostly very early in life. As no new understanding is obtained by considering this border condition while the complexity of the model description is increased substantially, we preferred not to describe it any further, for clarity's sake. However, our numerical results as well as all the graphs do incorporate these rare cases.

5) The optimal behavior is the one associated with the highest expected reproductive value. The fitness function $F(X, Y, t, d)$ corresponds to the expected reproductive value determined by the optimal behavior. This function predicts the mean number of eggs that 
the female, characterized by state $(X, Y, t, d)$, is expected to lay if she behaves in an optimal manner. If we therefore have the fitness value at a given time, we can calculate the expected reproductive values at the previous time step. The model is thus solved from the final time step $(36,26)$, to the first time step $(1,1)$. Successive backward iterations are used to deduce fitness values and the optimal decisions at each time step and for each value of $X$ and $Y$. The fitness value at time $(36,26)$ is therefore needed to solve the algorithm. Since the last time step is the maximum life span of an individual, its fitness values $F(X, Y, 36,26)$ at death is therefore necessarily 0 , regardless of the values of $X$ and $Y$.

In the model including resorption, females are allowed to resorb some of their mature eggs, and we added a new state variable, $R$, corresponding to the number of eggs in the process of being resorbed (Fig. 1). Regardless of how the female decides to treat the next host, she can assign $k$ eggs to resorption at the beginning of each time step, provided that $k$ is smaller than or equal to the number of mature eggs available. Thus, in addition to the host-handling decisions considered here, the number $k$ of eggs entering the resorption process at each new time step is taken into account. The possible decisions are denoted $1_{k}$ or $2_{k}$, with $\{1,2\}$ corresponding to host-feeding or oviposition. For example, $1_{4}$ would indicate host-feeding with the resorption of four eggs. When a female chooses to resorb a given number of eggs, they go from the mature egg category $(X)$ to the category of eggs undergoing resorption $(R)$. At each time step, a fraction $\delta$ of eggs in this category is resorbed and supplies some energy toward the reserves. A conversion factor $\gamma$ is applied to this energy in order to account for the energy loss associated with the process of resorption.

Equations of the model.-For the active period, the model calculates the change in variables for each possible decision (left-hand side of the equations) and the values of reproductive variables. Let us denote $r, x$, and $y$, the values of the state variables $R, X$, and $Y$ at time $(t, d)$. If the female decides to resorb $k$ eggs, the number of eggs undergoing resorption becomes $(r+k)$.

If no host is encountered, the variables evolve as follows:

$$
\begin{aligned}
& y_{0 k}(t+1, d)=y(t, d)-Y_{\mathrm{m}}+\gamma[r(t, d)+k] \delta \\
& x_{0 k}(t+1, d)=x(t, d)+\alpha-k \\
& r_{0 k}(t+1, d)=r(t, d)+k-[r(t, d)+k] \delta .
\end{aligned}
$$

If the female finds a host and decides to feed on it, host-feeding lasts two time steps. Therefore, processes of egg maturation, resorption, and maintenance costs update state variables accordingly. We assumed that the female can only decide to resorb eggs at the beginning of the first time step. With an energy gain of $Y_{\mathrm{h}}$, we have:

$$
\begin{aligned}
& y_{1 k}(t+2, d)=y(t, d)+Y_{\mathrm{h}}-2 Y_{\mathrm{m}}+2 \gamma[r(t, d)+k] \delta \\
& x_{1 k}(t+2, d)=x(t, d)+2 \alpha-k \\
& r_{1 k}(t+2, d)=r(t, d)+k-2[r(t, d)+k] \delta .
\end{aligned}
$$

If the female finds a host and decides to use it for oviposition,

$$
\begin{aligned}
& y_{2 k}(t+1, d)=y(t, d)-Y_{\mathrm{m}}+\gamma[r(t, d)+k] \delta \\
& x_{2 k}(t+1, d)=x(t, d)+\alpha-1-k \\
& r_{2 k}(t+1, d)=r(t, d)+k-[r(t, d)+k] \delta .
\end{aligned}
$$

Maximization process is a two-step process. The decision to resorb is taken at the beginning of each time step, before decisions related to host use.

The optimal number of eggs to resorb when seeking a host for feeding and the associated fitness value are determined according to

$$
\begin{aligned}
& F_{\mathrm{h}-\text { feed }}(x, y, r, t, d) \\
& =\max _{k}\left\{(1-\lambda)(1-\mu) F\left(x_{0 k}, y_{0 k}, r_{0 k}, t+1, d\right)\right. \\
& \left.\quad+\lambda(1-\mu)^{2} F\left(x_{1 k}, y_{1 k}, r_{1 k}, t+2, d\right)\right\} .
\end{aligned}
$$

The first factor represents the fact that no host is encountered and the second that a host is encountered. The host-feeding decision covers two time steps and thus fitness gains should be discounted with two probabilities of survival.

The optimal number of eggs to resorb when seeking a host for oviposition and the associated fitness value are determined according to

$$
\begin{aligned}
& F_{\text {ovip }}(x, y, r, t, d) \\
&=\max _{k}\left\{(1-\lambda)(1-\mu) F\left(x_{0 k}, y_{0 k}, r_{0 k}, t+1, d\right)\right. \\
&\left.+\lambda(1-\mu)\left[1+F\left(x_{2 k}, y_{2 k}, r_{2 k}, t+1, d\right)\right]\right\} .
\end{aligned}
$$

An oviposition adds 1 to the fitness value.

Finally, the highest fitness value and the associated optimal behavior are determined in maximizing over the host-handling decisions:

$$
\begin{aligned}
& F(x, y, r, t, d) \\
& \quad=\max _{\text {decision }}\left\{F_{\mathrm{h}-\text { feed }}(x, y, r, t, d), F_{\text {ovip }}(x, y, r, t, d)\right\} .
\end{aligned}
$$

The next procedure in the program consists in linking the last time step of the active period $(36, d)$ to the first of the following day $(1, d+1)$ by the mean of the sequential coupling method. The whole night is discounted for in a single operation covering 108 time steps. The female is only able to resorb and mature eggs. 
If she decides to resorb eggs, she does it using only those eggs present at the beginning of the night. During this period, the variables evolve as follows:

$$
\begin{aligned}
& y_{k}(1, d+1)=y(36, d)-Y_{\mathrm{m}} t_{\mathrm{n}}+t_{\mathrm{n}} \gamma[r(36, d)+k] \delta \\
& x_{k}(1, d+1)=x(36, d)-k+t_{\mathrm{n}} \alpha \\
& r_{k}(1, d+1)=r(36, d)+k-t_{\mathrm{n}}[r(36, d)+k] \delta .
\end{aligned}
$$

The optimal number of eggs to resorb and the fitness are determined according to

$$
F(x, y, r, 36, d)=\max _{k}(1-\mu)^{t_{n}} F\left(x_{k}, y_{k}, r_{k}, 1, d+1\right) .
$$

The dynamics between these time steps are processed as between any other time step. There is no gradual decline in the number of eggs to resorb during a night and the model cannot be used for analyzing processes occurring within a night. Females obtain their acquired energy reserves right at the beginning of the next step, early in the morning. According to our assumption of a linear decrease of the mass of resorbing eggs, the modeling of egg resorption follows the simple rule $r(t)$ $=k\left[1-\delta\left(t-t_{0}\right)\right]$, with $t_{0}$ being the time step in which the $k$ eggs are put in resorption. As the SDP works backward, however, we lack values for both $k$ and $t_{0}$ during the active period, and this equation cannot therefore be used. This problem can however be dealt with linear interpolation. It induces some statistical variability, but no bias in the behavioral decisions.

Forward iterations. - Once the optimal strategy was determined through backward iteration, the models were used for successive Monte Carlo iterations forward in time. This procedure can be used to simulate individual trajectories for monitoring and quantifying changes in physiological variables, behavior, and lifehistory traits in the females from a cohort (Clark and Mangel 2000). The female begins life with a level $Y_{\max }$ of energy reserves and an egg load of $0-2$ (D. Giron, personal communication). At each time step, a number $z$ between 0 and 1 is selected at random from a uniform distribution. If $z \leq \mu$, the female dies. If $\mu<z \leq \mu+\lambda$ (1 $-\mu$ ), the female survives and encounters a host. If $z>\mu$ $+\lambda(1-\mu)$, no host is encountered. When the female is required to make a behavioral decision (when she encounters a host or needs to resorb eggs), it is assumed that the decision made is optimal as a function of state variables, this optimality being determined by the dynamic programming model. The data extracted for the different sets of parameters correspond to simulations for $n=5000$ females.

\section{Estimation of parameters}

The parameters described here are summarized in Table 1. We ensured that the parameters used matched the experimental conditions of a previous study (Casas et al. 2005), facilitating direct comparisons with the experimental data obtained in this previous study.
Time horizon and survival function.-Each active period is divided into 10 -minute time steps. The duration of the active period is fixed at $6 \mathrm{~h}$, giving $t_{\mathrm{a}}=$ 36 time step, and an inactive period of $18 \mathrm{~h}\left(t_{\mathrm{n}}=108\right)$. The females live a maximum of $D=26$ days. This is the maximum life span observed when females have access to sufficient hosts for feeding (Casas et al. 2005). Rejection or oviposition lasts $<10$ minutes and therefore lasts one time step in the models. This procedure implies that there is no time cost of oviposition (Rosenheim 1999a, Ellers et al. 2000). We adopted this procedure since we believe that such considerations are of secondary importance for the questions we are focusing on. By contrast, host-feeding takes $>10$ minutes, the mean duration of this process being 17.3 minutes (Giron et al. 2004). We therefore rounded so that host-feeding lasts two time steps in our models. The insects were raised in the laboratory, thus extrinsic causes of mortality were very limited. Nevertheless, we considered the background mortality according to a standard approach (Heimpel et al. 1998). The probability of extrinsic mortality $\mu$ corresponds to the day on which half the parasitoids have already died: $\ln (2) / \mu=15$ days, and thus in one time step $\mu=\ln (2) /(15 \times 144)=0.0003$.

Egg maturation and resorption.-A female lays a mean of 39 eggs over a period of 14.25 days (Casas et al. 2005). If all the mature eggs are laid, the mean egg number matured each day is 2.74 eggs, the number of time steps in a day is $144\left(t_{\mathrm{a}}+t_{\mathrm{n}}=36+108=144\right)$, and thus the number of eggs matured in one time step is $\alpha=39 /(14.25 \times 144)=0.02$ eggs. The time required for egg resorption is generally estimated at less than a day in hymenopterans (Jervis and Kidd 1986). We therefore used a value corresponding to this order of magnitude, 100 time steps, or 1000 minutes $(16.67 \mathrm{~h})$, as the default parameter. Assuming a constant rate of resorption, a fraction $\delta=1 \%$ of each egg undergoing resorption is thus resorbed in one time step. Like many anhydropic (producing yolk-rich eggs) hymenopteran parasitoid species, Eupelmus vuilleti can store no more than two mature eggs per ovariole (R. Richard, personal observation). As females possess six ovarioles, we therefore considered 12 to be the maximum possible mature egg load $X_{\max }$. The same considerations applied to the maximum number of eggs undergoing resorption, $R_{\max }$, which also cannot exceed 12 . In addition, the cumulative number of mature eggs and eggs undergoing resorption cannot exceed $12([x+r] \leq$ 12). Furthermore, the simultaneous occurrence of resorption in all the ovarioles prevents oviposition (Jervis and Kidd 1986). Resorption generally begins with the terminal egg (King and Richards 1968). We therefore imposed an additional constraint: oviposition becomes impossible if six or more eggs are undergoing resorption.

Value of energy parameters.-Energy values for capital reserves, host-feeding $\left(Y_{\mathrm{h}}\right)$, and the energy content of an egg were deduced from the data presented 

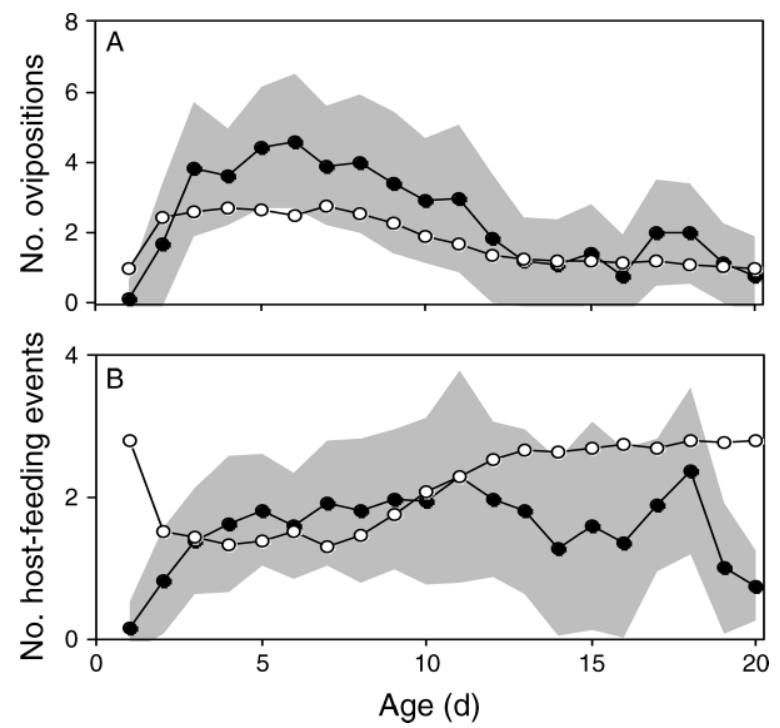

FIG. 2. Observed and predicted daily number of (A) ovipositions and (B) host-feeding events in relation to age of the hymenopteran parasitoid Eupelmus vuilleti. Experimental data are represented with solid circles (mean \pm SD shown by the gray area); values predicted by the dynamic programming model with resorption are represented with open circles for the parameter values of $\lambda=0.12$ (probability of host encounter in one time step) and $\alpha=0.02$ (number of eggs matured in one time step).

by Casas et al. (2005). Each energy value was converted into kilojoules and is expressed in egg equivalents. The value for capital reserves used was fixed as the maximum threshold of energy reserves $\left(Y_{\max }\right)$. The value used in the model is estimated from the difference between the available energy measured at emergence and that measured at the time of death of the females. The female therefore dies when the level of reserves reaches the critical threshold $Y_{\text {crit }}$ of 0 . Energy costs for maintenance in one time step (which includes the energy costs for egg production and the energy contained in the eggs) are grouped together in the term $Y_{\mathrm{m}}$. This term is calculated from the mean energy acquired by each female, through capital reserves and host-feeding, divided by mean life span. Thus, $Y_{\mathrm{m}}=\left(Y_{\max }+\right.$ $\left.Y_{\mathrm{h}} n_{\mathrm{h}}\right) /(14.25 \times 144)=(120+5.22 \times 21.7) /(14.25 \times$ $144)=0.114$ egg equivalent in one time step, with $n_{\mathrm{h}}=$ mean number of host-feeding events during the life of a female. A conversion factor $\gamma=0.7$ was applied to the energy acquired by host-feeding, to give the energy available to the female, taking into account digestion yield (McNeill 1999, McDonald et al. 2002). The value $Y_{\mathrm{h}}$ given takes this yield into account. Similarly, this factor was used to describe the energy lost during the recuperation of energy content of an egg by resorption.

\section{RESUlts}

We first determined whether the complete model fitted experimental data, by comparing the mean daily number of ovipositions and host-feeding events predicted by the stochastic dynamic programming (SDP) model with those obtained experimentally (Casas et al. 2005). We then investigated the biological mechanisms underlying host-use strategies through a study of host-feeding. The analytical model allowed us to identify the biological phenomena underlying the decision-making process. Analysis of the SDP model without resorption made it possible to quantify the relative impact of each of these mechanisms on the decision-making process, as a function of the conditions encountered by the females. We then described the conditions favorable for resorption and analyzed the effect of including resorption in the model on the predicted host management strategies adopted by the females. Finally, we used forward simulations to describe how the changes in strategies are reflected in the behavior displayed, based on the complete model with resorption.

\section{Validation of the model}

Experimental data and the output of the model showed mean daily number of ovipositions to be highest at the start of life (days 2-8), and declining thereafter (Fig. 2). Probability of host encounter is estimated from the mean number of hosts used (parasitized or consumed) per day and by females as reported by Casas et al. (2005), giving a mean probability of host encounter of $\lambda=0.12$ in one time step. The prediction of the complete SDP model (with resorption) was good as it fell within the margin of error for almost all days. A slightly higher number of eggs matured in one time step ( $\alpha=0.032)$ combined with a slightly higher probability of host encounter $(\lambda=0.17)$ increased the predicted number of oviposition events at the start of life to the observed level.

The daily number of host-feeding events predicted by the model also fell largely within the margin of error for the experimental data. However, two divergences were noted. Firstly, the number of host-feeding events predicted by the model was too high for the first day. This deviation results from the starting point of the model: at emergence, individuals have few or no eggs (between 0 and 2) and the females therefore rapidly experience egg limitation, resulting in the hosts initially encountered being used for host-feeding. The markedly lower daily number of host-feeding events observed experimentally on days 1 and 2 clearly reflects a biological phenomenon not taken into account by our model, probably linked to a time lag between emergence and complete maturity. Secondly, although the number of host-feeding events predicted by the model remained largely within the margin of error, the two curves diverged after day 10 . The outputs of the model suggested that there should be an increase in the number of host-feeding events after this time point, but no such increase was observed in the experimental data. These results are, however, compatible with the experimental data, as shown in the Discussion. 


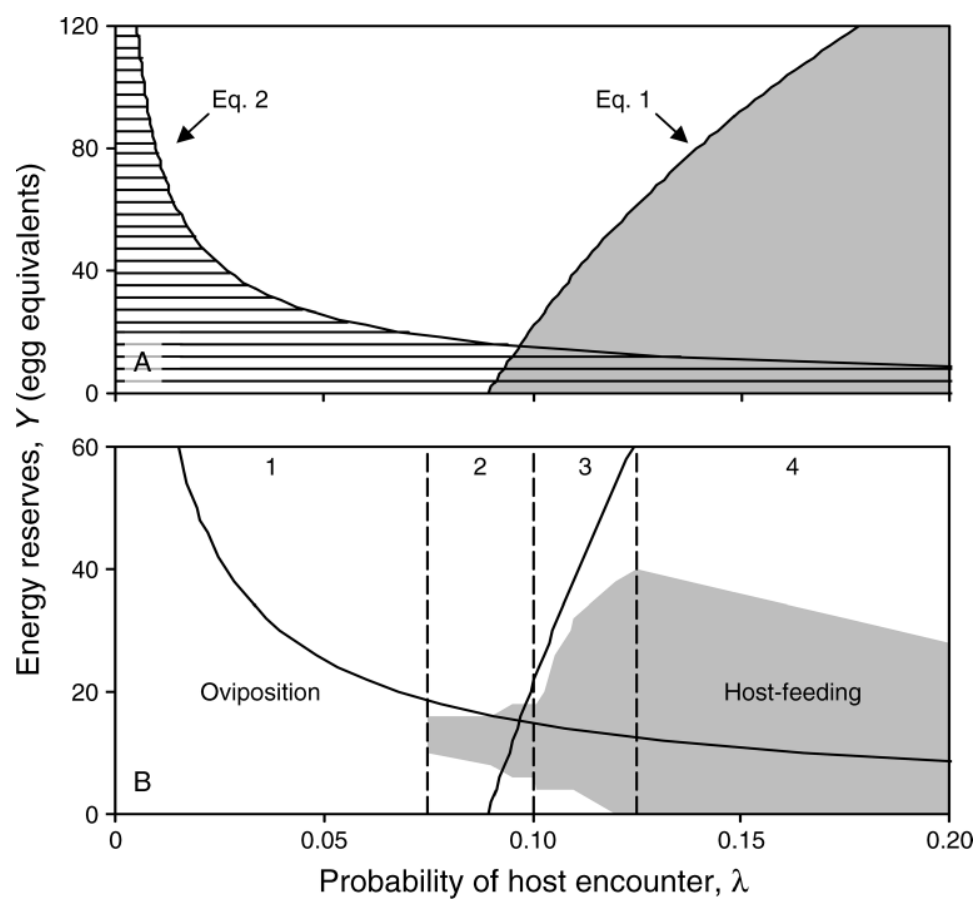

FIG. 3. (A) State space and decision zones from the analytical model, and (B) superimposed on the decision zones defined by the dynamic programming model without resorption. (A) Host-feeding does not add fitness benefits to the left of the line defined by Eq. 1. It is beneficial to the right of that line (gray area). The likelihood of death through starvation is higher than the likelihood of extrinsic mortality below the line defined by Eq. 2 (hatched area); it is reversed above that line. Parameter values of the dynamic programming model are $d=5$ (day of life), $t=18$ (time steps during active period), and $x(t, d)=2$. (B) The four zones (1-4) delimitated by vertical dotted lines refer to increasing probabilities of encounters with hosts and are used in the Results section.

\section{Analytical model of host-feeding}

This part of the paper deals with the analytical hostfeeding model. We aimed here to identify the biological phenomena and trade-off affecting decision-making variables of host-feeding.

Eq. 1 of the analytical model, which includes the predicted number of supplementary encounters per hostfeeding event, divides the $(\lambda, Y)$ space into two parts (Fig. 3A). On the left of the curve of Eq. 1 (not shaded in gray), host-feeding provides no gain in fitness. On the right of the curve, host-feeding is beneficial. This result may be interpreted as a function of the projected life span of the female. On the left of the curve, host-feeding does not increase the number of hosts available for oviposition by prolonging the life of the female. In this case, we will define the strategy of host use adopted by the female as "short term," as it involves the use of hosts exclusively for immediate reproduction through oviposition. To the right of the curve, host-feeding makes it possible to increase the number of hosts available for oviposition. The female thus adopts a strategy of host use and energy-resource management, making it possible to maximize the number of oviposition events over her entire potential life span. This strategy may therefore be described as "long term."

A comparison of the mean number of hosts encountered per day with the number of hosts required to compensate metabolic costs supports these intitial conclusions. Females need to feed on a mean 3.14 hosts per day to compensate the energy requirements for metabolic maintenance in an exact fashion. This mean number of consumed hosts corresponds to the mean number of hosts encountered at a probability of host encounter of 0.087 . At lower probability of host encounter, females are unlikely to meet suficient hosts to compensate for energy losses. When the probability of host encounter is slightly greater than 0.087 , there may be enough hosts to compensate for these costs, but the female cannot lay any eggs if she consumes all these hosts. It is therefore only when the mean number of hosts encountered is markedly greater that the female can both compensate for metabolic costs by feeding and lay her eggs, allowing a long-term host-use strategy.

Eq. 2 defines a certain threshold at which the probability of dying from starvation before encountering a host equals the extrinsic probability of mortality (Fig. 3A). In the parts of the graph below the curve of Eq. 2 (hatched areas), the probability of dying from starvation is higher than the extrinsic probability of mortality. With females being more subjected to starvation risk in this zone, we might expect a higher propensity to host-feed. Above the curve, the inverse is true, with the probability of death from starvation being lower than the extrinsic probability of mortality. This situation leads to the female laying eggs, because her 
chances of dying from another cause are greater than those of dying from starvation and she has the possibility of counterbalancing the possible risk of starvation by subsequently feeding.

The superimposition of these two curves divides the $(\lambda, Y)$ space into four approximate areas (Fig. 3A). Based on the two equations of the model, females finding themselves in the upper left-hand area (white) will choose to lay eggs, whereas those in the lower righthand area will choose to feed on the host (hatched gray). In the other two areas, the different equations result in opposite conclusions. In the lower left-hand area (hatched white), despite the female having a greater probability of dying from starvation than from other causes, the model predicts that host-feeding will provide no gain of fitness, regardless of the conditions, because the prolongation of life by host-feeding cannot compensate for the loss of oviposition. In this part of the space, oviposition is therefore favored. In the upper right-hand area (gray), host-feeding provides a gain of fitness, leading females to invest in future rather than immediate reproduction. However, the risks of starvation are lower than the risk of extrinsic mortality, favoring immediate reproduction. These two phenomena thus oppose each other in the upper-right area, and the analytical model cannot give a clear conclusion concerning the outcome of this conflict. A more precise determination of the costs and benefits of the two decisions requires an analysis of the results obtained with our dynamic programming model. This also frees the analytical model of two major constraints, making it possible to take into account the possibility of feeding repeatedly rather than only once, and with daily periodicity rather than assuming a constant rate of encounters during both night and day.

\section{Stochastic dynamic programming (SDP) host-feeding model}

Consistent with results classically obtained, the SDP model without resorption initially indicated that, for a given probability of host encounter, host-feeding is favored by decreases in energy reserves. A similar, but less marked result was obtained for the effect of egg load (data not shown): host-feeding is largely favored when the female has only one egg available. However, egg load has only a slight effect, decreasing the energy threshold for feeding if the female has two or more eggs available. This effect varies little with age, other than decreasing toward the maximum life span.

The range of values for energy reserves over which the female should feed, according to the SDP model without resorption, is shown in Fig. 3B. The parameter values used in this figure facilitate comparison with the analytical model, and a change in these values has little effect on the results. In this example, the values presented are those for day 5, chosen arbitrarily, although we avoided values above day 8 because few females live longer than this when probability of host encounter $(\lambda)$ is less than 0.10 . The data correspond to the middle of the day $(t=18)$, because certain phenomena linked to the diurnal/nocturnal cycle make it more difficult to interpret the overall strategy adopted by the female toward the beginning or end of the day. Egg load was fixed at two, based on the considerations just described.

According to the SDP model without resorption, the females feed in the zone corresponding principally to the gray-shaded area of the analytical model. This tendency varies with probability of host encounter, and four different zones may be visually distinguished (Fig. 3B). At very low probability of host encounter $(\lambda<0.075$, zone 1), no host-feeding occurs unless egg load reaches 0 . At low probability of host encounter $(0.075 \leq \lambda \leq$ 0.10 , zone 2), host-feeding is favored over a narrow range of energy reserve values. The tendency to feed increases strongly at intermediate probability of host encounter $(0.10<\lambda \leq 0.125$, zone 3$)$. The tendency to feed, having reached a maximum, then gradually decreases $(\lambda>0.125$, zone 4$)$.

The feeding zone observed in zone 2 results is a particular case linked to the cycling between day and night, involving a short-term strategy. A single hostfeeding event brings enough energy to live for longer than a foraging period, 45 time steps exactly. If a female has barely enough energy to live through the night, she might therefore live another foraging period by adding up the energy gained through that host-feeding. This enables a female to live and die at the end of the second foraging period, or in the following night, in contrast to the situation where she would avoid host-feeding and would die within the first night (data not shown). By extending their life by one foraging period, these females increase their fitness by a large margin, explaining why host-feeding is favored. A similar but inverse explanation holds for the bottom portion in zone 3, for which the SDP predicts no host-feeding, in contrast to the analytical model. In this case, the extra gain from hostfeeding benefits survival during the night only, without supplemental ovipositions.

The abrupt increase in the threshold for feeding in zone 3 for the SDP model corresponds to that observed during the study of the analytical model and results from the mechanism incorporated in Eq. 1 (Fig. 3B). In this zone, the prolongation of life by host-feeding makes it possible to expect more than a host encounter in the future and thus to increase the reproductive success of females. We are therefore in a situation in which the female adopts a long-term strategy. However, we found that the threshold for feeding diverged from the one predicted by Eq. 1 when probability of host encounter exceeded 0.11 . The curve in zone 3 corresponds to a composite curve determined by processes given in Eqs. 1 and 2 .

In zone 4, the threshold for feeding in the SDP model gradually decreases and qualitatively follows a trajectory similar to the curve of Eq. 2 in the analytical model. In 


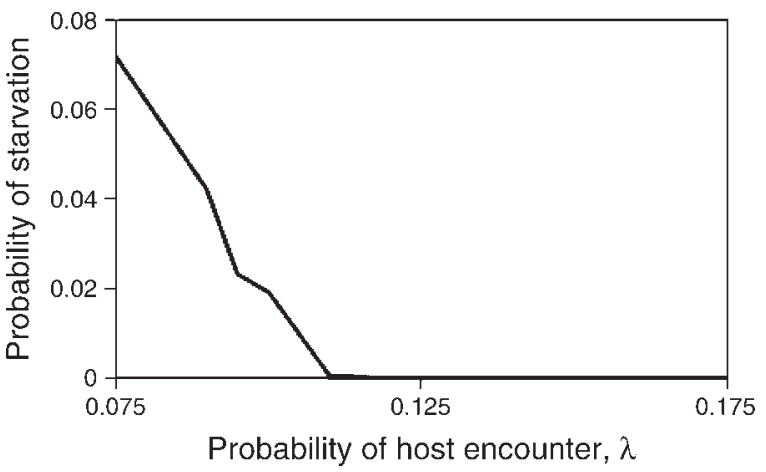

FIG. 4. Predicted likelihood of death through starvation before encountering a host, as a function of probability of host encounter, given that energy reserves equate to the upper hostfeeding threshold determined by the dynamic program without resorption (Fig. 3).

this zone, changes in the threshold for feeding therefore reflect a compromise reached by balancing the risks of death by starvation against those of extrinsic mortality. When probability of host encounter exceeds 0.11 and energy reserves reach the threshold for feeding of the SDP model, the probability of dying from starvation is very close to 0 (between $10^{-5}$ and $10^{-6}$; Fig. 4). Since the risks of starvation are very low, females therefore prefer to oviposit and postpone host-feeding, keeping the energetic threshold under which they feed as low as possible.

The decision to feed on a host depends therefore essentially on the two basic mechanisms described by the analytical model. The SDP model can be used to quantify the influence of each of these mechanisms in the upper right-hand area of Fig. 3A (gray). At probabilities of host encounter comprised between 0.10 and 0.11 , the extra ovipositions made possible by hostfeeding (Eq. 1 of the analytical model) determine greatly the location of the feeding threshold of the SDP model. The same equation also predicts that host-feeding should take place at high probabilities of host encounter, but is unable to determine the extent to which hostfeeding is favored. At probabilities of host encounter exceeding 0.125 , the feeding threshold of the SDP model follows a trajectory similar to the curve of Eq. 2, but remains above the threshold predicted by this equation, indicating that the risks of starvation remain much smaller than those of extrinsic mortality.

The mean lifetime number of host-feeding events as a function of probability of host encounter, as determined by forward simulations, reflects the results obtained (Fig. 5). At low probability of host encounter, the number of host-feeding events remains low and relatively constant. The number of host-feeding events increases abruptly at intermediate probabilities of host encounter $(\lambda>0.10)$ and is maintained at a high level at high probability of host encounter $(\lambda>0.15)$. The transition between short-term and long-term strategies accounts for the abrupt increase in the number of host-

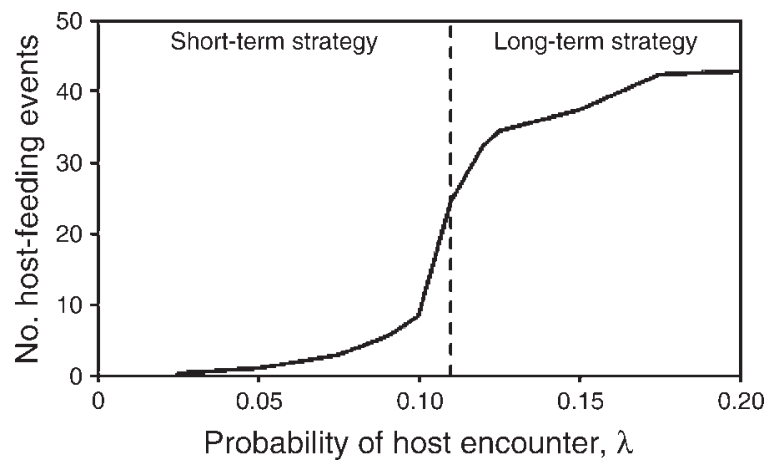

FIG. 5. Lifetime number of host-feeding events predicted by the dynamic program without resorption, as a function of probability of host encounter. The vertical dashed line indicates the probability of host encounter at which females change strategy.

feeding events. We consider that this change in strategy occurred at a probability of host encounter of 0.11 , corresponding to a mean of 3.96 hosts encountered per day, as the number of host-feeding events suddenly became much greater at this probability of encountering a host (Fig. 5).

\section{Resorption}

The SDP model with resorption showed that resorption does not generally occur before day 7 (Fig. 6). Resorption has a major impact at very low probabilities of host encounter, but its impact is greatest at intermediate probabilities of host encounter (Fig. 7). The number of eggs resorbed is maximal at a probability of host encounter of 0.10 , at which point females may resorb up to 13.8 eggs, on average, during the mean life span (Fig. 7). The gain from resorption is maximal at a probability of host encounter of 0.11 , at which point females lay a mean of 2.4 extra eggs, corresponding to a $10.4 \%$ increase in laying (Fig. 7). The existence of local maxima at very low and intermediate probabilities of host encounter suggests that two distinct phenomena may be at work.

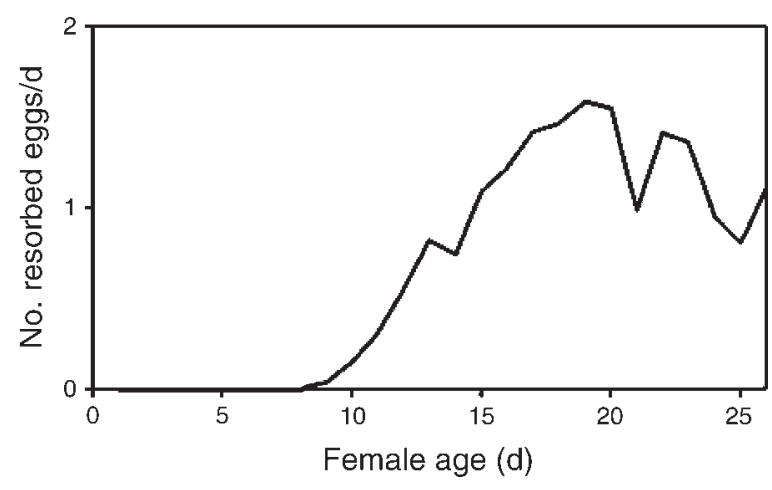

FIG. 6. Predicted number of daily resorbed eggs, as a function of female age. $\lambda=0.11$. 


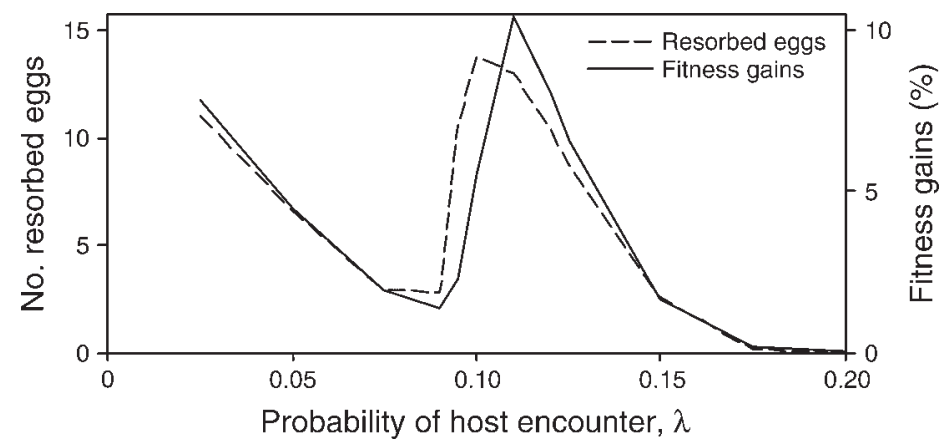

FIG. 7. Predicted lifetime number of resorbed eggs (dashed line) and fitness gains obtained through resorption (solid line), as a function of probability of host encounter.
At very low probability of host encounter, the benefits of resorption are evident: egg production exceeds the number of hosts encountered, so the eggs are resorbed to provide energy to compensate for maintenance costs. The resulting prolongation of life allows the females to meet more hosts and, therefore, to lay more eggs. At high probability of host encounter $(\lambda \geq 0.15)$, resorption provides little or no gain because the frequency of host encounters makes it possible for the female both to feed to compensate for the energy costs of maintenance and to lay the eggs produced. In this case, resorption should be avoided because the resorbed egg can no longer be laid, thus representing a loss to oviposition. The gain in fitness is maximal at intermediate probabilities of host encounter. It is also apparent that once females have adopted a long-term strategy, the number of realized ovipositions increases faster with the probability of host encounter, in both the presence and absence of resorption (Fig. 8). Females displaying resorption change strategy when the probability of host encounter exceeds 0.095 (determined based on the same indicators as for the SDP model without resorption). Females not displaying resorption opt for a long-term strategy when probability of host encounter exceeds 0.11 . As the strategy change occurs at a lower probability of host encounter in the presence of resorption, the benefits of resorption increase as long as the females without resorption have not also adopted a long-term strategy, accounting for the maximal gain at a probability of host encounter of 0.11 . In conclusion, resorption leads to a gain of fitness because it allows females to adopt a longterm strategy at lower probabilities of host encounter. We investigated the role of resorption in this change in strategy in more detail, by investigating how a change to a long-term strategy at low probability of host encounter affects resource acquisition by females. When females without resorption change strategy $(\lambda=0.11)$, the probability of finding enough hosts in a day to compensate for daily maintenance costs is about 0.97 (Fig. 9). By maintaining a sufficiently high threshold for host-feeding, females can counterbalance the risks of starvation and should also mostly find enough additional hosts each day for oviposition. However, when females displaying resorption change strategy $(\lambda=$ $0.095)$, this probability falls to 0.66 . The daily acquisi- tion of energy by host-feeding may therefore frequently be insufficient. Resorption necessarily compensates for this irregularity. These conclusions raise questions about why resorption makes it possible to adopt a long-term strategy at lower probabilities of host encounter and how it helps to compensate for subsequent irregularities in energy acquisition. These questions are discussed in detail in the Discussion.

\section{Lifetime and daily host-handling decisions}

We have described two distinct foraging strategies as a function of the probability of meeting hosts and the biological mechanisms underlying these changes. We now describe the effects of these changes in strategy on the life history of the females, the behavior they adopt, and the trajectories of their physiological variables, using the results of the complete SDP model with resorption. Fig. 10 illustrates the consequences of these changes, using examples of energy-reserves dynamics and decision-making variables at low probability of host encounter $(\lambda=0.09$, Fig. 10A) and at intermediate probability of host encounter $(\lambda=0.125$, Fig. $10 \mathrm{~B})$.

In both cases, the females begin their lives with large energy reserves, such that in the early part of their lives, these reserves are maintained above the threshold for host-feeding (Fig. 10A and short-term strategy zone in Fig. 10B). The females experiencing different probabilities of host encounter thus behave in a similar manner: they favor oviposition, with host-feeding occurring only

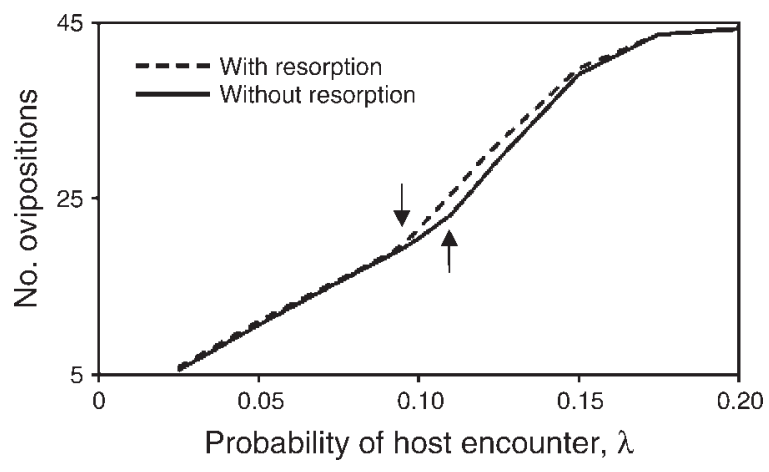

FIG. 8. Predicted lifetime number of ovipositions, without (solid line) and with (dashed line) resorption. Arrows indicate the points where there is a change of strategies. 
FIG. 9. Likelihood of encountering enough hosts per day in order to compensate for energy costs, as a function of probability of host encounter. Arrows indicate the change of strategies. The zones defined by dashed lines are those shown in Fig. 3.

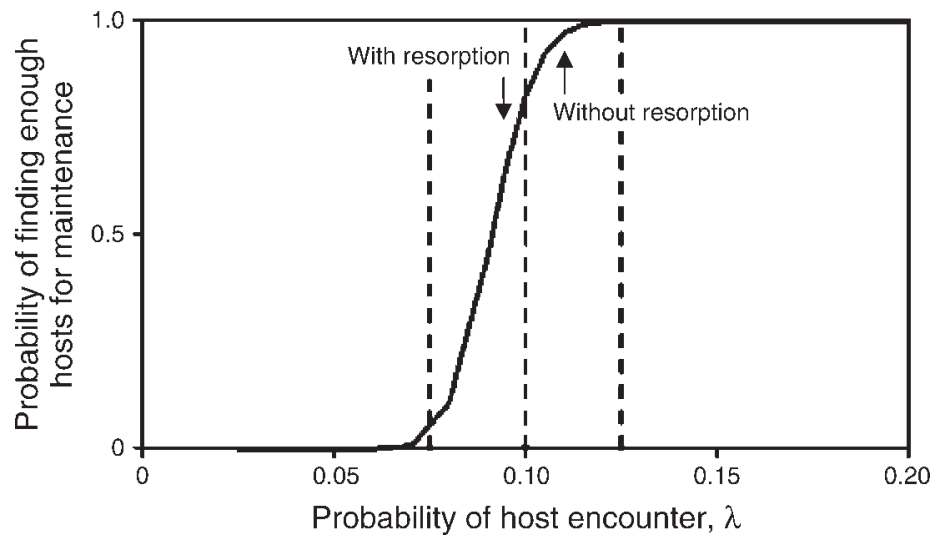

when they have no more eggs to lay. As each female begins the day with a minimum of two eggs, corresponding approximately to the number of eggs matured during the night, the egg load is depleted only after a certain number of encounters. The proportion of females host-feeding therefore gradually increases during the active period (Fig. 11).

Females faced with low probability of host encounter do not change strategy later in life. As the number of hosts encountered does not allow them to compensate for daily metabolic costs and the females adopt a shortterm strategy, reserves inevitably decline. When reserves fall to the threshold for feeding, they are no longer maintained and the females soon die of starvation. At intermediate probability of host encounter (Fig. 10B long-term strategy zone), the range of values over which the females feed is large because the females adopt a long-term strategy, the number of hosts encountered being sufficient to compensate for daily metabolic costs. The females begin the day with energy reserves below the threshold for feeding, as a large amount of energy is consumed during the night. They then feed until their reserves exceed this threshold, laying eggs thereafter. In this situation, the diurnal pattern of behavior is thus reversed: most females feed early in the day and the proportion of females laying eggs increases during the course of the day (Fig. 11). In this case, the females generally do not lay all the eggs they produce,

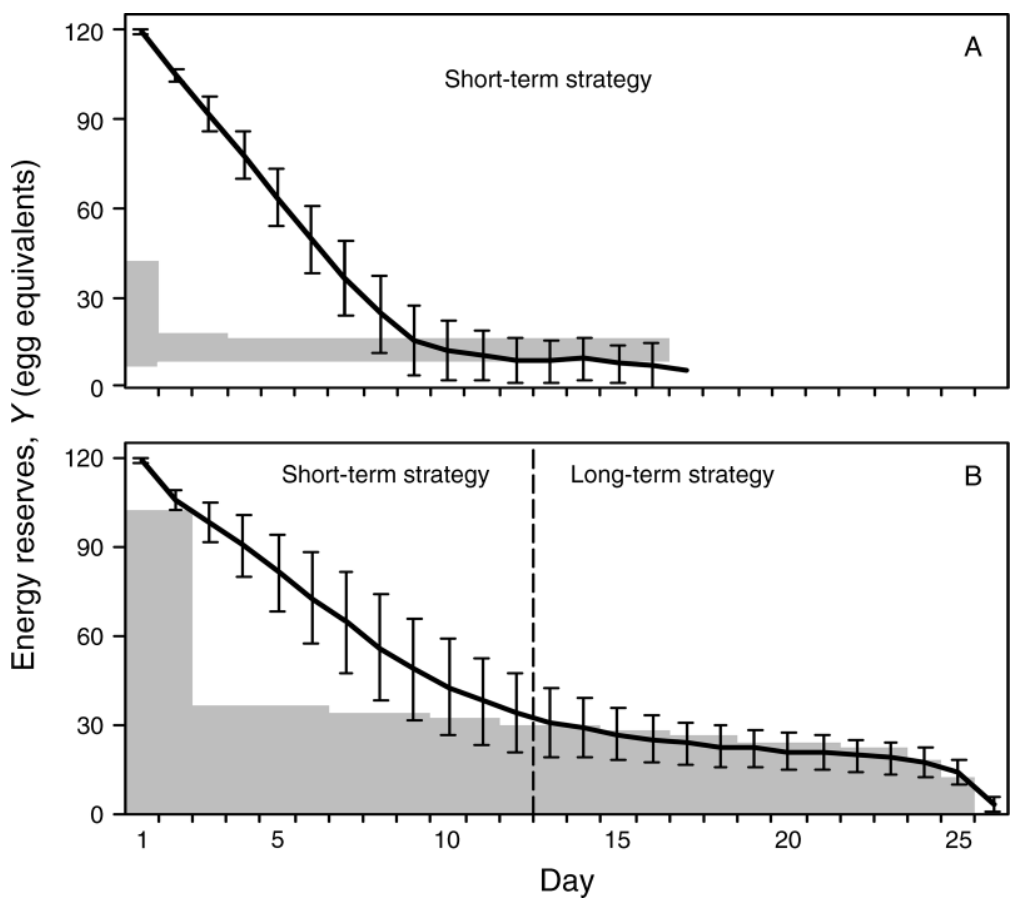

FIG. 10. Predicted dynamics of energy reserves (lines, with mean \pm SD) and zone of host-feeding decision (gray zones), as a function of (A) low $(\lambda=0.09)$ and $(B)$ intermediate $(\lambda=0.125)$ probability of host encounter and female age, from the dynamic program with resorption. The feeding zones are defined using the mean egg load of females at time step $t=18$. The dashed line in panel (B) divides the life span of a female into the short-term strategy and long-term strategy periods. 


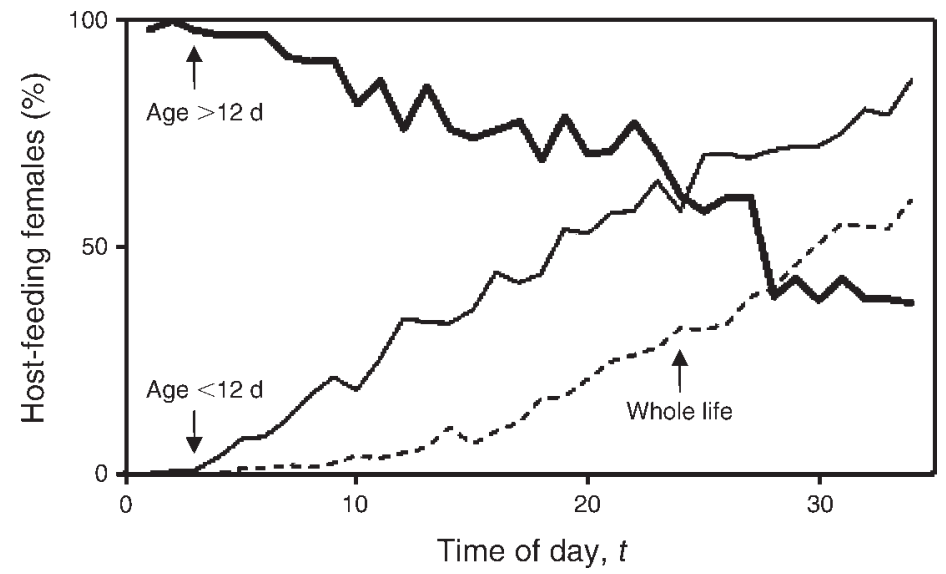

FIG. 11. Percentage of host-feeding females, as a function of the time of the day, female age, and for two probabilities of host encounter. At intermediate probability of host encounter $(\lambda=$ $0.125)$, females change behavior during their lives (thin line, young females; bold line, older females). At low probability of host encounter $(\lambda=0.09)$, female behavior remains constant over the entire lifetime (dashed line). particularly if the probability of host encounter is below 0.15 . Excess eggs are thus produced and are available for resorption. It is at this time in the life of the female that behavioral changes relating to the adoption of a longterm strategy become apparent.

\section{Discussion}

\section{Assumptions of the model}

Here, we return to some of the most important hypotheses and analyze in more detail the differences observed between the output of the complete model and observational data. Our model assumes a constant rate of egg maturation. However, egg production has been shown to be dynamic, responding in particular to variations in host quality and abundance and behavioral choices (Papaj 2000, Casas et al. 2009). Our model thus underestimates the plasticity of egg production. A variable egg production rate avoids the need to invest more energy than necessary in this process. Resorption, as considered by our model, plays a similar role, as it makes it possible to recover excess energy investment in eggs. It is therefore possible that the apparent benefits of resorption through such a mechanism would have been smaller if our model had taken into account a variable rate of egg maturation, because more energy could have been saved by limiting egg production rather than recovering excess energy through resorption. However, the model developed by Ellers et al. (2000) does predict that the number of eggs produced exceeds the number of expected encounters with hosts in most cases, because it is preferable to invest excess energy in egg production rather than to miss opportunities to lay eggs (responding to a priori considerations). Resorption, by giving to the females the possibility to recover energy from eggs, allows thereby females to invest more eggs in reproduction than the expected number of host encounters, since energy is still available when needed (responding a posteriori to actual conditions). We also assumed that egg maturation and resorption can occur concurrently. We made this assumption because of the lack of relevant data concerning these processes for Eupelmus vuilleti.
Egg maturation and resorption processes occurring concurrently has indeed been reported for other parasitic wasps (e.g., Flanders 1942, Edwards 1954, Lloyd 1966), and such phenomena occur in the normal ovarian activity of numerous other insect species belonging to different insect orders (see references in Bell and Bohm 1975).

In the absence of other limitations, the individuals in our simulations could theoretically live and produce eggs indefinitely, thanks to host-feeding, if they adopted a long-term strategy. Recent studies of the acquisition and allocation of nutrients have shown that certain nutrients acquired during larval development are replaced ineffectively or not at all by host-feeding (Rivero et al. 2001, Casas et al. 2005, Strand and Casas 2007, Jervis et al. 2008). This is the case for lipids, which have been shown to be limiting for the fecundity of $E$. vuilleti (Giron et al. 2004). Lipid allocation may be variable, depending in particular on carbohydrate availability (Strand and Casas 2007), but lipid reserves are likely to be exhausted long before the end of the maximum life span of the organism in our model (day 26). The existence of nutrients that cannot be replaced by hostfeeding in adult insects thus limits the time period during which the female can hope to survive and lay eggs, potentially reducing, at least partly, the benefits of the long-term strategy predicted by our model.

The divergences between our model and experimental data for the number of host-feeding events per day may be accounted for as follows: in E. vuilleti, host-feeding increases both the longevity and fecundity of individuals (Giron et al. 2004, Casas et al. 2005). Based on the finding that females live only seven days in the absence of food, Giron et al. (2004) assumed that the amount of energy acquired from host-feeding must increase after seven days. This increase was observed, not through an increase in the frequency of feeding, but through an increase in the duration of each host-feeding event, which was itself correlated with the amount of energy acquired. The amount of energy acquired per hostfeeding event was considered constant in our model. This model therefore predicted an increase in the total 

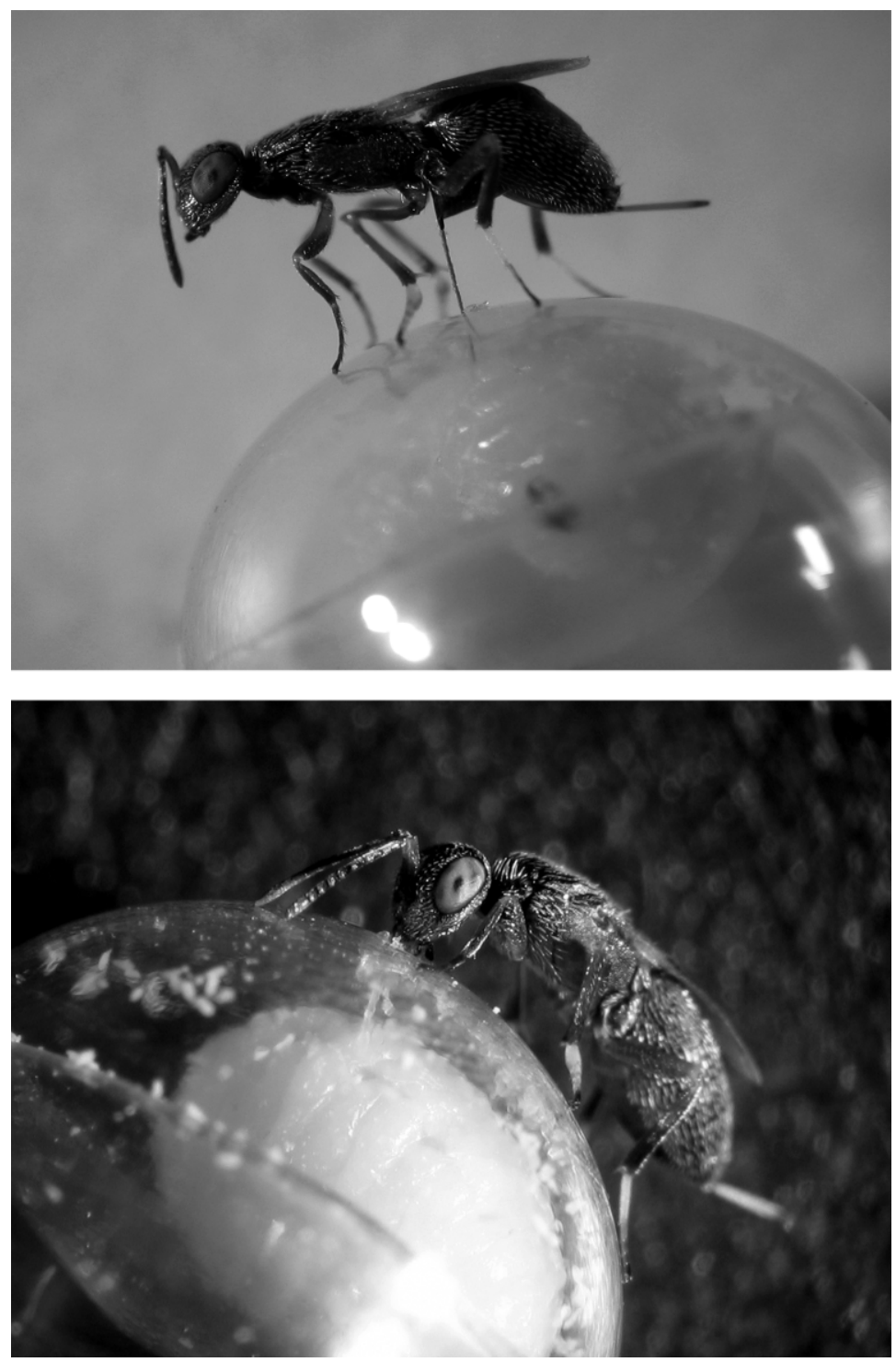

Plate 1. (A) Eupelmus vuilleti (CRW) (Hymenoptera: Eupelmidae) is a tropical solitary host-feeding ectoparasitoid of third- to fourth-instar larvae of Callosobruchus maculatus (F.) (Coleoptera: Bruchidae) infecting Vigna unguiculata (Fabaceae) pods and seeds. Females are synovigenic, i.e., they are born with some immature eggs and need to feed from the host in order to sustain egg production and maturation. In this species, females feed from the host by puncturing its cuticle and creating a feeding tube with secretions from their ovipositor. (B) The females then turn and use the feeding tube to extract the host fluids with their mouthparts. Prior to those photographs, hosts had been extracted from the seeds and placed inside a gelatin capsule. This system allowed the observation of feeding behavior and the extraction of the host-feeding fluids from the host-feeding tube. Photo credits: David Giron.

amount of energy obtained from host-feeding at about this age through an increase in the frequency of feeding (Fig. 2B). Such changes in energy acquisition through feeding during the life of an organism are well documented for many species of insects (Bernays and Chapman 1972, 1994, Bernays and Simpson 1982, Chapman and de Boer 1995, Chapman 1998). The increase in the frequency of feeding induces a decrease in the rate of oviposition, because the hosts used for feeding cannot be used for oviposition. In general, the allocation of more effort to the acquisition of energy results in a decrease in oviposition activity. This change in the rate of oviposition as a function of the age of the individual is frequently observed in insects (Partridge et al. 1987, Boggs 1997, Novoseltsev et al. 2002). These changes may be attributed, at least in our case, to the exhaustion of capital reserves (Giron and Casas 2003). When a female reaches a certain age, her energy reserves are exhausted, reaching a zone in which host-feeding is more favored (Fig. 10). The female then adopts a longterm strategy, resulting in the changes in the frequency of feeding and oviposition observed. 
Unfortunately, several striking results from the model cannot be compared due to a lack of experimental data: the two peaks of gain from resorption and the reversal of preference between host-feeding and oviposition concomitant to the change of foraging strategy. In fact, we failed to find any but one longitudinal study of behavioral decisions over the entire parasitoid lifetime as function of host density (Burger et al. 2006), and very few longitudinal studies with parasitoids in general (Heimpel et al. 1997). The situation was identical fifteen years ago, when McNamara et al. (1994) published their seminal theoretical study on foraging routines of a small bird in winter: there was a lack of empirical data to provide a test of their theory. Nevertheless, their study spurred a whole sequence of refined experiments and adjusted models making "the small bird in winter" one of the best understood cases of optimality applied to behavioral ecology (Brodin 2007). A similar effort is needed to understand foraging routines in parasitoids, and we hope that our model will encourage the collection of observations and design of experimental tests.

\section{Host-feeding strategies: \\ short-term vs. long-term strategies}

The change in the likelihood of host-feeding with changes in probability of host encounter predicted by our models is similar to that obtained with other models of host-feeding or searching for food in parasitoids (Chan and Godfray, 1993, Collier 1995b, Heimpel and Collier 1996, Sirot and Bernstein 1996, Bernstein and Jervis 2007). In general, these models, like ours, predict that the organism favors immediate reproduction if the availability of hosts or food is low. The tendency to feed is also maximal at intermediate probabilities of host encounter and the transition between the strategies adopted at low and intermediate probabilities of host encounter is sometimes abrupt. Once this transition has occurred, the tendency to feed decreases at higher probabilities of host encounter because energy resources are more easily renewed. These models thus predict qualitatively similar behavior. However, none of these models provides a mechanistic approach to the reasons for this change in host-feeding frequency nor explores the consequences of these changes in strategy for behavior and life history traits.

The abrupt transition between approaches takes place at an intermediate probability of host encounter, which acts as a threshold separating the two distinct approaches: short-term and long-term. If probability of host encounter is below the transition threshold, the number of hosts encountered is too small to cover food needs to compensate for energy losses and to keep the females alive over long periods and is also too small to compensate for losses of oviposition opportunities through future reproduction. In these conditions, host-feeding takes place only in the case of egg-load depletion. Above the transition threshold, the frequency of encounters with hosts is high enough to compensate for daily metabolic costs and to extend the life of individuals. Host-feeding is therefore favored, because the loss of opportunities for oviposition is compensated for by the prolongation of life by host-feeding, increasing the total number of opportunities to lay eggs (Charnov and Stephens 1988). Oviposition thus occurs less frequently, but over a longer period. Once this long-term strategy has been adopted, the tendency to feed on hosts is determined by the balance between the risks of death from starvation and those of extrinsic mortality. In this case, the organism maintains its energy reserves at a level high enough to minimize the risk of death from starvation. When reserves exceed the threshold, the parasitoid can postpone host-feeding and maximize its reproductive success through immediate reproduction.

\section{The link between host-feeding and resorption}

Natural selection results in a reproductive strategy in which the number of eggs produced is adapted to match the number of hosts encountered (Rosenheim 1996). However, the stochastic nature of the environment makes a perfect match between these two variables impossible. As a result, the reproductive success of adult females may be limited by the number of hosts encountered (time limitation) or by the number of eggs available for oviposition (egg limitation) (Rosenheim 1996, 1999b, Sevenster et al. 1998, Papaj 2000). As encounters with hosts cannot be predicted with certainty, the species concerned have evolved a high degree of flexibility in terms of physiological and behavioral traits (Jervis et al. 2001, 2008). This flexibility enables them to manage resources in a dynamic manner, with females able to modify the amounts of resources used for immediate and future reproduction, irrespective whether these resources are expressed as energy, eggs, time, or hosts. Synovigeny allows females to adjust their reproductive efforts to variations in the frequency of encounters with hosts by modifying the amount of energy invested in egg production (Ellers et al. 2000, van Baalen 2000, Jervis et al. 2001, Ellers and Jervis 2004, Casas et al. 2009). As reproduction is spread over a longer period in synovigenic species, it is not surprising that hostfeeding and resorption are frequently found in such species, making it possible to adjust the balance between immediate and future reproduction. Indeed, Jervis et al. (2001) demonstrated in a large comparative study that there was a strong link between synovigeny, hostfeeding, and resorption. Host-feeding and resorption are observed mostly in synovigenic species, although exceptions have been found. Most of the species displaying resorption practice host-feeding, although, again, there are exceptions. Nevertheless, to our knowledge, all parasitoid species displaying resorption have an alternative source of food during adulthood. For example, Leptomastix dactylopii and Phanerotoma franklini do not display host-feeding, but feed on 
honeydew or nectar, respectively, while undergoing resorption (Lloyd 1966, Rivero-Lynch and Godfray 1997, Sisterton and Averill 2002). Thus, these conclusions can be transposed to parasitoid species feeding on carbohydrate-rich substrates, in which the compromise between feeding and immediate reproduction is expressed in terms of the allocation of time and energy in different patches, rather than in terms of host utilization (Bernstein and Jervis 2007).

Consistent with the classical view of resorption, our model effectively predicts large gains from this process when there are very few opportunities for reproduction and feeding. Nevertheless, by associating resorption with periods of nutrient stress and considering this process as a "last resort" strategy, as is commonly done, one underestimates the adaptive value of resorption. By contrast, the maximal gain from resorption predicted by our model occurs at an intermediate probability of host encounter. Through resorption, females develop a long-term strategy at lower probabilities of host encounter than they would otherwise be able to deal with in the absence of resorption. There is therefore a range of values, at intermediate probabilities of host encounter, over which only females with resorption adopt a long-term strategy. Opting for a long-term strategy involves more host-feeding and thus, females displaying resorption feed on hosts more frequently than those without resorption, paradoxical though this may seem at first glance. The adaptive potential of resorption is maximal at intermediate probabilities of host encounter, but only in the presence of an alternative source of nutrients. The adoption of a long-term strategy implies the optimization of reproduction over long periods, requiring greater flexibility in resource allocation. In conclusion, resorption is favored because it responds to the need for flexibility when host-feeding is possible.

\section{Resorption as a buffer against stochasticity}

For the adoption of a long-term strategy to be beneficial, female survival must be ensured for long enough to surpass the benefits she would have gained from a short-term strategy. The adoption of a long-term strategy therefore involves a certain risk. The management of this risk requires stochastic factors linked to host encounters to be taken into account. The irregularity of these factors may make it impossible for females to lay eggs every day, and their cumulative effects may lead to death from starvation. The intensity of extrinsic mortality probably also counterbalances the possible benefits of adopting a long-term strategy (Heimpel and Collier 1996, Heimpel et al. 1997, Jervis et al. 2001).

Resorption modifies the perception of the starvation risk by females, which then favor a long-term strategy in more hostile conditions. The explanation of these findings requires consideration of the ways in which energy is acquired by the adult. After emergence, the females of this species have only two ways of gaining additional energy: host-feeding and resorption. Our model focused solely on the energetic aspects of resorption, so its benefits could only lead to survival gains, however minimal. However, the amount of energy released by resorption is probably not the key factor, as shown by Casas et al. (2005). The moment at which this energy is used may instead be the key factor, as suggested by Rosenheim et al. (2000). Resorption is indeed under the full control of females. It is also modeled as a deterministic process, so that a resorbing female knows the exact timing of her future energy gains from resorption. By contrast, the probability of host encounter is not under the control of females. Worst, because it is a probability, a female cannot know the timing of next encounters with precision. Resorption is an internal, homeostatic feedback mechanism which maintains biological conditions against external disturbances and vagaries (Aström and Murray 2008). Thus, despite its low energy gains, resorption is a buffer against stochasticity.

Before extending our results to other foraging behavior in other animals, we summarize here the advances made by this study. This work explains the rapid change of propensity to host-feed as a function of host density, an observation commonly made but hardly understood; identifies a marked change of horizon in foraging strategies during the lifetime of parasitoids, with several striking implications for daily behavioral routines; provides for the first time an evolutionary explanation for the costly process of egg resorption; and discloses the hitherto unexpected deep relationships between two sources of nutrients. Our results can furthermore be generalized by establishing certain analogies between resorption and other foraging behavior in other, unrelated animals. Hoarding behavior, observed in certain birds and mammals, involves storing energetic foods in hiding places for later use (short-term hoarding; Smith and Reichman 1984, Källander and Smith 1990, Brodin 2007). Models developed to explore the adaptive value of this behavior, which is striking in terms of the discrepancy between the resources hidden and those actually used, suggest that the food stored in this way provides more of an insurance policy against stochastic events rather than a real source of energy, just like resorption (Hitchcock and Houston 1994, Brodin 2007). By allowing individuals to adopt more risky strategies, hoarding increases fitness, even if the stores are not used (Brodin 2000, 2007). This analogy also shows that the most controllable resources are not necessarily within the animal; they may be part of the external environment. Certain birds, such as the acorn woodpeckers (Koenig and Mumme 1987, Hitchcock and Houston 1994), accumulate spectacular quantities of acorns, which they bury into the bark of trees, thereby controlling their food availability and decreasing the starvation risks associated with foraging for rarer resources. Thus, host-feeding and resorption are highly 
complementary activities (as are hoarding and active food searching) due to the degree with which each resource can be controlled and varied. The homeostatic adaptive value of resorption, like that of hoarding, can only be understood in the context of the existence of an alternative source of nutrients. Host-feeding provides a lot of energy, but this income is conditioned on finding a host, which is stochastic and not controllable. Resorption, by contrast, provides much less energy but is an income which is deterministic and under full control of the animal.

\section{ACKNOWLEDGMENTS}

We thank D. Giron, C. Magal, S. Pincebourde, and one referee for useful comments on previous versions of the manuscript. We also thank J. Rosenheim and the second referee, L. Hemerik, for particularly incisive and astute comments and for the care with which they scrutinized previous versions of the manuscript. This work is based on the Master's thesis of R. Richard, carried out at the University of Tours. We thank the University of Tours and CNRS for the financial support needed to complete the work.

\section{Literature Cited}

Asplen, M. K. 2007. Life history correlates in female parasitoid wasps: exploring old concepts and new frontiers. Dissertation. University of Arizona, Tucson, Arizona, USA.

Aström, K. J., and R. M. Murray. 2008. Feedback systems. Princeton University Press, Princeton, New Jersey, USA.

Bell, W. J., and M. K. Bohm. 1975. Oosorption in insects. Biological Review 50:373-396.

Bernays, E. A., and R. F. Chapman. 1972. Meal size in nymphs of Locusta migratoria. Entomologia Experimentalis et Applicata 15:399-410.

Bernays, E. A., and R. F. Chapman. 1994. Host-plant selection by phytophagous insects. Chapman and Hall, New York, New York, USA.

Bernays, E. A., and S. J. Simpson. 1982. Control of food intake. Advances in Insect Physiology 16:59-118.

Bernstein, C., and M. A. Jervis. 2007. Food-searching in parasitoids: the dilemma of choosing between "intermediate" or future fitness gains. Pages 129-171 in E. Wajnberg, C. Bernstein, and J. van Alphen, editors. Behavioral ecology of insects parasitoids. Blackwell, London, UK.

Boggs, C. L. 1997. Dynamics of reproductive allocation from juvenile and adult feeding: radiotracer studies. Ecology 78 : 192-202.

Brodin, A. 2000. Why do hoarding birds gain fat in winter in the wrong way? Suggestions from a dynamic model. Behavioral Ecology 11:27-39.

Brodin, A. 2007. Theoretical models of adaptive energy management in small wintering birds. Philosophical Transactions of the Royal Society B 362:1857-1871.

Burger, J. M. S., L. Hemerik, J. C. van Lenteren, and L. E. M. Vet. 2004. Reproduction now or later: optimal host-handling strategies in the whitefly parasitoid Encarsia formosa. Oikos 106:117-130.

Burger, J. M. S., Y. Huang, L. Hemerik, J. C. van Lenteren, and L. E. M. Vet. 2006. Flexible use of patch-leaving mechanisms in a parasitoid wasp. Journal of Insect Behavior 19:155-170.

Casas, J., S. Pincebourde, N. Mandon, F. Vannier, R. Poujol, and D. Giron. 2005. Lifetime nutrient dynamics reveal simultaneous capital and income breeding in a parasitoid. Ecology 86:545-554.

Casas, J., F. Vannier, N. Mandon, J. P. Delbecque, D. Giron, and J. P. Monge. 2009. Mitigation of egg limitation in parasitoids: immediate hormonal response and enhanced oogenesis after host use. Ecology 90:537-545.

Chan, M. S. 1991. Host-feeding in parasitic wasps: a study of population patterns generated by individual behaviour. Dissertation. Imperial College, University of London, London, UK.

Chan, M. S., and H. C. J. Godfray. 1993. Host-feeding strategies of parasitoid wasps. Evolutionary Biology 7:593604.

Chapman, R. F. 1998. The insects: structure and function. Fourth edition. Cambridge University Press, Cambridge, UK.

Chapman, R. F., and G. de Boer. 1995. Regulatory mechanism in insect feeding. Chapman and Hall, New York, New York, USA.

Charnov, E. L., and D. W. Stephens. 1988. On the evolution of host selection in solitary parasitoids. American Naturalist 132:707-722.

Clark, C. W., and M. Mangel. 2000. Dynamic state variable models in ecology. Oxford University Press, New York, New York, USA.

Collier, T. R. 1995a. Host-feeding, egg maturation, resorption and longevity in the parasitoid Aphitis melinus. Annals of the Entomological Society of America 88:206-214.

Collier, T. R. 1995b. Adding physiological realism to dynamic state variable models of parasitoid host-feeding. Evolutionary Ecology 9:217-235.

Eckelbarger, K. J. 1994. Diversity of metazoan ovaries and vitellogenic mechanisms: implications for life history theory. Proceedings of the Biological Society of Washington 107: 193-218.

Edwards, R. L. 1954. The effect of diet on egg maturation and resorption in Mormoniella vitripennis (Hymenoptera, Pteromalidae). Quartertly Review of Microscopical Sciences 95: 459-468.

Ellers, J., and M. A. Jervis. 2004. Why are so few parasitoid wasp species pro-ovigenic? Evolutionary Ecology Research 6: 993-1002.

Ellers, J., J. G. Sevenster, and G. Driessen. 2000. Egg load evolution in parasitoids. American Naturalist 156:650-665.

Flanders, S. E. 1942. Oösorption and ovulation in relation to oviposition in the parasitic Hymenoptera. Annals of the Entomological Society of America 35:251-266.

Gauthier, N., and J. P. Monge. 1999. Behavioural and physiological responses to conflicting oviposition stimuli in a synovigenic parasitoid. Physiological entomology 24:303310 .

Giron, D., and J. Casas. 2003a. Lipogenesis in an adult parasitic wasp. Journal of Insect Physiology 49:141-147.

Giron, D., and J. Casas. 2003b. Mothers reduce egg provisioning with age. Ecology Letters 6:273-277.

Giron, D., S. Pincebourde, and J. Casas. 2004. Lifetime gains of host-feeding in a synovigenic parasitic wasp. Physiological Entomology 29:436-442.

Giron, D., A. Rivero, N. Mandon, E. Darrouzet, and J. Casas. 2002. The physiology of host-feeding in parasitic wasps: implications for survival. Functional Ecology 16:750-757.

Godfray, H. C. J. 1994. Parasitoids. Behavioral and evolutionary ecology. Princeton University Press, Princeton, New Jersey, USA.

Grosch, D. S. 1950. Starvation studies with the parasitic wasp Habobracon. Biological Bulletin, Woods Hole 99:65-73.

Heimpel, G. E., and T. R. Collier. 1996. The evolution of hostfeeding behaviour in insect parasitoids. Biological Review 71: 373-400.

Heimpel, G. E., M. Mangel, and J. A. Rosenheim. 1998. Effects of time limitation and egg limitation on lifetime reproductive success in parasitoids. American Naturalist 152:273-289.

Heimpel, G. E., and J. A. Rosenheim. 1995. Dynamic host feeding by the parasitoid Aphytis melinus: the balance 
between current and future reproduction. Journal of Animal Ecology 64:153-167.

Heimpel, G. E., J. A. Rosenheim, and D. Kattari. 1997. Adult feeding and lifetime reproductive success in the parasitoid Aphytis melinus. Entomologia Experimentalis et Applicata 83:305-315.

Heimpel, G. E., J. A. Rosenheim, and M. Mangel. 1996. Egg limitation, host quality, and dynamic behavior by a parasitoid in the field. Ecology 77:2410-2420.

Hitchcock, C. L., and A. I. Houston. 1994. The value of a hoard: not just energy. Behavioral Ecology 5:202-205.

Houston, A. I., and J. M. McNamara. 1999. Models of adaptive behaviour. Cambridge University Press, Cambridge, UK.

Houston, A. I., J. M. McNamara, and H. C. J. Godfray. 1992. The effect of variability on host-feeding and reproductive success in parasitoids. Bulletin of Mathematical Biology 54: 465-476.

Jervis, M. A., J. Ellers, and J. A. Harvey. 2008. Resource acquisition, allocation and utilization in parasitoid reproductive strategies. Annual Review of Entomology 53:361385.

Jervis, M. A., G. E. Heimpel, P. N. Ferns, J. A. Harvey, and N. A. C. Kidd. 2001. Life-history strategies in parasitoid wasps: a comparative analysis of "ovigeny." Journal of Animal Ecology 70:442-458.

Jervis, M. A., and N. A. C. Kidd. 1986. Host-feeding strategies in hymenopteran parasitoids. Biological Reviews 61:395434.

Källander, H., and H. G. Smith. 1990. Food storing in birds: an evolutionary perspective. Current Ornithology 7:147-207.

King, P. E., and C. R. Hopkins. 1963. Length of life of the sexes in Nasonia vitripennis (Walker) (Hymenoptera: Pteromalidae) under conditions of starvation. Journal of Experimental Biology 40:751-761.

King, P. E., and J. G. Richards. 1968. Oösorption in Nasonia vitripennis (Hymenoptera: Pteromalidae). Journal of Zoology 154:495-516.

Koenig, W. D., and R. L. Mumme. 1987. Population ecology of the cooperatively breeding acorn woodpecker. Princeton University Press, Princeton, New Jersey, USA.

Lloyd, D. C. 1966. Some aspects of egg resorption in Leptomastix dactytlopii How. [Hymenoptera, Encyrtidae]. Entomophaga 11:365-381.

Mangel, M. 1989. Evolution of host selection in parasitoids: does the state of the parasitoid matter? American Naturalist 133:688-705.

McDonald, P., R. A. Edwards, J. D. F. Greenhalgh, and C. A. Morgan. 2002. Animal nutrition. Prentice Hall, Upper Saddle River, New Jersey, USA.

McNamara, J. M., A. I. Houston, and S. L. Lima. 1994. Foraging routines of small birds in winter: a theoretical investigation. Journal of Avian Biology 25:287-302.

McNeill, A. R. 1999. Energy for animal life. Oxford University Press, Oxford, UK.

Minkenberg, O. P. J. M., M. Tatar, and J. A. Rosenheim. 1992. Egg load as a major source of variability in insect foraging and oviposition behaviour. Oikos 65:34-142.

Novoseltsev, V. N., R. Arking, J. A. Novoseltseva, and A. I. Yashin. 2002. Evolutionary optimality applied to Drosophila experiments: hypothesis of constrained reproductive efficiency. Evolution 56:1136-1149.

Papaj, D. R. 2000. Ovarian dynamics and host use. Annual Review of Entomology 45:423-448.
Partridge, L., A. Green, and K. Fowler. 1987. Effects of eggproduction and of exposure to males on female survival in Drosophila melanogaster. Journal of Insect Physiology 33: 745-749.

Price, P. W. 1973. Reproductive strategies in parasitoid wasps. American Naturalist 107:684-693.

Rivero, A., and J. Casas. 1999a. Incorporating physiology into parasitoid behavioural ecology: the allocation of nutritional resources. Researches in Population Ecology 41:39-45.

Rivero, A., and J. Casas. 1999b. Rate of nutrient allocation to egg production in a parasitic wasp. Proceedings of the Royal Society of London B 266:1169-1174.

Rivero, A., D. Giron, and J. Casas. 2001. Lifetime allocation of juvenile and adult nutritional resources to egg production in a holometabolous insect. Proceedings of the Royal Society of London B 268:1231-1237.

Rivero-Lynch, A., and H. C. J. Godfray. 1997. The dynamics of egg production, oviposition and resorption in a parasitoid wasp. Functional Ecology 11:184-188.

Rivero, A., and A. West. 2005. The costs and benefits of host feeding in parasitoids. Animal Behaviour 69:1293-1301.

Roff, D. A. 1992. The evolution of life histories: theory and analyses. Chapman and Hall, New York, New York, USA.

Roff, D. A., and D. J. Fairbairn. 2008. The evolution of tradeoffs: where are we? Journal of Evolutionary Biology 20:443447.

Rosenheim, J. A. 1996. An evolutionary argument for egg limitation. Evolution 50:2089-2094.

Rosenheim, J. A. 1999a. Characterizing the cost of oviposition in insects: a dynamic model. Evolutionary Ecology 13:41165.

Rosenheim, J. A. 1999b. The relative contributions of time and eggs to the cost of reproduction. Evolution 53:376-385.

Rosenheim, J. A., G. E. Heimpel, and M. Mangel. 2000. Egg maturation, egg resorption and the costliness of transient egg limitation in insects. Proceedings of the Royal Society of London B 267:1565-1573.

Sevenster, J. G., J. Ellers, and G. Driessen. 1998. An evolutionary argument for time limitation. Evolution 52: 1241-1244.

Sirot, E., and C. Bernstein. 1996. Time sharing between host searching and food searching in parasitoids: state-dependent optimal strategies. Behavioral Ecology 7:189-194.

Sisterton, M. S., and A. L. Averill. 2002. Costs and benefits of food foraging for a braconid parasitoid. Journal of Insect Behavior 15:571-588.

Smith, C. C., and O. J. Reichman. 1984. The evolution of food caching by birds and mammals. Annual Review of Ecology and Systematics 15:329-351.

Strand, M. R., and J. Casas. 2007. Parasitoid and host nutritional physiology in behavioural ecology. Pages 113128 in E. Wajnberg, C. Bernstein, and J. van Alphen, editors. Behavioral ecology of insects parasitoids. Blackwell, London, UK.

Stearns, S. C. 1992. The evolution of life histories. Oxford University Press, Oxford, UK.

van Baalen, M. 2000. The evolution of parasitoid egg load. Pages 103-120 in M. Hochberg and A. R. Ives, editors. Parasitoid population dynamics. Princeton University Press, Princeton, New Jersey, USA.

Wheeler, D. 1996. The role of nourishment in oogenesis. Annual Review of Entomology 41:407-431.

Williams, G. C. 1966. Natural selection, the costs of reproduction, and a refinement of Lack's principle. American Naturalist 100:687-690. 